\title{
The white matter module-hub network of semantics revealed by semantic
}

\section{dementia}

Martina Sundqvist*1,2,3,4,5,6, Alexandre Routier*7,1,2,3,4 Bruno Dubois $^{1,2,3,4,9}$, Olivier Colliot $^{8,2,3,4,7}$, Marc Teichmann ${ }^{1,2,3,4,9}$

* denotes equal contribution

${ }^{1}$ Institut du Cerveau et de la Moelle épinière, ICM, FrontLab, F-75013, Paris, France

${ }^{2}$ Inserm, U 1127, F-75013, Paris, France

${ }^{3}$ CNRS, UMR 7225, F-75013, Paris, France

${ }^{4}$ Sorbonne Université, F-75013, Paris, France

${ }^{5}$ Université Paris-Saclay, AgroParisTech, INRAE

${ }^{6}$ Institut Curie - PSL Research University, Translational Research Department, Breast Cancer Biology Group, 26 rue d'Ulm, 75005 Paris, France

${ }^{7}$ Inria, Aramis project-team, F-75013, Paris, France

${ }^{8}$ Institut du Cerveau et de la Moelle épinière, ICM, F-75013, Paris, France

${ }^{9}$ AP-HP, Institut de la mémoire et de la maladie d'Alzheimer, Centre de Référence

“Démences Rares”, Hôpital de la Pitié-Salpêtrière, Paris, France

\section{Correspondence to:}

Marc Teichmann

Department of Neurology, National Reference Center for « rare dementias »

Hôpital de la Pitié Salpêtrière. 47-83, boulevard de l'Hôpital. 75013 Paris. France.

E-mail: marc.teichmann@psl.aphp.fr. Telephone: +331421675 34. Fax: +33142167504

\section{Disclosures}

No author reports disclosures relevant to this study and manuscript. 


\begin{abstract}
Cognitive neuroscience exploring the architecture of semantics has shown that coherent supramodal concepts are computed in the anterior temporal lobes (ATL) but it is unknown how/where modular information implemented by posterior cortices (word/object/face forms) is conveyed to the ATL hub. We investigated the semantic module-hub network in healthy adults $(\mathrm{N}=19)$ and in semantic dementia patients $(\mathrm{N}=28)$ by combining semantic assessments of verbal and non-verbal stimuli and MRI-based fiber tracking using seeds in three module-related cortices implementing i) written word forms ('Visual Word Form Area'), ii) abstract lexical representations (posterior-superior temporal cortices), and iii) face/object representations ('Face Form Area').
\end{abstract}

Fiber tracking revealed three key tracts linking the ATL with the three module-related cortices. Correlation analyses between tract parameters and semantic scores indicated that the three tracts subserve semantics, transferring modular verbal or non-verbal object/face information to the left and right ATL, respectively. The module-hub tracts were functionally and microstructurally damaged in semantic dementia whereas damage to non-module specific ATL tracts (inferior longitudinal fasciculus, uncinate fasciculus) had more limited impact on semantic failure.

These findings identify major components of the white matter module-hub network of semantics and they corroborate/materialize claims of cognitive models positing direct links between modular and semantic representations. In combination with modular accounts of cognition, they also suggest that the currently prevailing 'hub-and-spokes' model of semantics could be extended by incorporating an intermediate module level containing invariant representations, in addition to 'spokes' which subserve the processing of a near-unlimited number of sensori-motor and speech-sound features.

Keywords: Semantic dementia, semantics, diffusion MRI, tractography 


\section{Introduction}

A core issue of cognitive neuroscience is to understand how and where the meaning of words, faces and objects of the surrounding world is extracted and combined into coherent concepts. Much work has been done to identify the cortical regions underpinning semantics and most authors currently advocate that the implementation of abstract concepts is subtended by a supramodal hub situated in the anterior temporal lobes (ATL) (Patterson et al., 2007; Rice et al., 2015; Lambon Ralph et al., 2017). However, it is largely unknown how modular information, such as for example word forms or face and object representations implemented by remote brain modules, is conveyed to the anterior temporal hub. The currently prevailing 'hub-andspokes' model of semantics, which is grounded on connectionist accounts, states that the socalled 'spokes', located mostly in posterior brain regions, represent sensory/speechsound/motor features that aliment the ATL hub (Lambon Ralph et al., 2017) via white matter connections (e.g., Binney et al., 2012; Guo et al., 2013; Rice et al., 2015). However, sensorymotor features are 'complex' and variable (Lambon Ralph et al., 2017), and the 'hub-andspokes' model hardly accounts for an important line of cognitive research having demonstrated that highly variable features of for example speech sounds are concatenated into invariant modular language representations (word forms) stored in the mental lexicon (Fodor, 1983; Caramazza and Miozzo 1997; Levelt, 1999; Indefrey and Levelt 2004; Pinker and Jackendoff, 2005; Tyler et al., 2005; Ullman, 2001). Such invariant modular representations (modules) might provide robust and exploitable information for the supra-modal semantic hub which attributes meaning to word forms. The same holds for object representations that need to be extracted from highly variable signals of the surrounding world, and which are stored in an object repertory/lexicon (sometimes called 'structural description system'; Lloyd-Jones and Humphreys, 1997; Gerlach et al., 1999). An important issue is therefore to explore whether 
there are, in addition to connections with 'spokes', white matter connections that directly link the ATL hub with cortical regions involved in cognitive modules.

Semantic Dementia (SD), affecting selectively semantic processing in its early stage, has initially allowed for the identification of the crucial role of the ATL (Patterson et al., 2007) and it might be an appropriate model for investigating the unsolved module-hub network issue. SD is a neurodegenerative condition characterized by multi-modal semantic deficits surfacing with single-word comprehension deficits, anomia, and face and object recognition difficulties (Gorno-Tempini et al., 2011). It is currently integrated into the spectrum of primary progressive aphasias (PPA) as the 'semantic variant of PPA' (Gorno-Tempini et al., 2011). However, the terminology 'primary progressive aphasia' can be misleading given the multi-modal semantic failure and we will therefore use the more integrative disease label, i.e. SD. SD is associated with bilateral damage to the ATL, which is left-predominant in early stages, including both cortical and subcortical structures of this region (Mummery et al., 2004; Agosta et al., 2010). Multiple anatomo-functional studies on SD have stressed the link between ATL atrophy and breakdown of semantics (Bozeat et al., 2000; Snowden et al., 2004; Mion et al., 2010; Mesulam et al., 2013; Iaccarino et al., 2015), indicating that the ATL constitute a bilaterally distributed hub underlying semantic computations which might bind modality-specific or modular information into coherent supra-modal concepts (Lambon Ralph et al., 1999; Patterson et al., 2007; Binney et al., 2012; Lambon Ralph et al., 2017). This view was also strengthened by investigations using inhibitory transcranial stimulation over the ATL inducing multi-modal semantic impairment in healthy adults (Pobric et al., 2007; 2010). Accordingly, a meta-analysis of functional MRI and PET investigations in healthy adults has shown that semantic tasks independently from the modality of stimuli input (verbal or non-verbal) generated bilateral activation of the ATL cortices (Rice et al., 2015). However, such research did not explore how modular information is conveyed to the semantic hub, and whether subcortical fiber 
connections linking specific brain modules to the ATL are involved in semantic processes. Furthermore, it is unclear whether such connections might play a core role regarding the distinct processing of verbal versus non-verbal/object information given that several authors, criticizing the bilateral multi-modal view, have attributed this distinction directly to left versus right ATL roles (Snowden et al., 2004; Gainotti, 2013; Mesulam et al., 2013).

The existence of multiple connections to the ATL dedicated to semantics is suggested by numerous findings indicating a semantic network including multiple cortical areas. For example, Damasio et al. (2004) have shown that semantic tasks such as picture naming depend, in addition to the ATL, on remote cortical regions such as left posterior inferior cortices of the temporal lobe. Likewise, Binder et al. (2009) and Vandenberghe et al. (1996; 2013) have identified various cortical regions, such as the left ventral occipital-temporal junction, the left superior temporal gyrus, the left middle temporal gyrus and the left inferior frontal gyrus, contributing to verbal and non-verbal semantics. Several of these non-ATL regions are involved in modular representations of words or faces or visual objects. In particular, the mid-posterior region of the right fusiform gyrus is dedicated to the representation/processing of faces and the cortical region has been referred to as the 'fusiform face area' (FFA) (Kanwisher et al., 1997). The FFA and its vicinity have subsequently been shown to be also critical for the processing of well-known visual objects other than faces (Gerlach et al., 1999; Gauthier et al., 2000; Tarr and Gauthier, 2000; Xu, 2005). Regarding language, the left mid/posterior region of the fusiform gyrus has been reported to selectively underpin the representations of written words and the cortical region has been referred to as the 'visual word form area' (VWFA) (Cohen et al., 2002; McCandliss et al., 2003). In addition, several authors have demonstrated that posterior parts of the left superior/middle temporal cortices subtend more generally lexical representations of both phonological and orthographic word forms (Kotz et al., 2002; Graves et al., 2007; 2008; Race and Hillis, 2015). However, the anatomo-functional connectivity between such modular 
cortical regions and the ATL has not been explored given that previous research was mostly anatomically-driven. This research has identified ATL connections to remote regions, which are not involved in modular language/object/face representations but in the processing of sensory-motor features such as the inferior longitudinal fasciculus (ILF) linking the ATL and the occipital lobe (Catani et al., 2003). Likewise, several authors have shown connections with a variety of posterior regions some of which probably implement sensory-motor features referred to as the 'spokes' by the 'hub-and-spokes' model (e.g., Binney et al., 2012; Guo et al., 2013). In the same vein, more anterior frontal regions such as the lateral/medial inferior frontal cortex which connects with the ATL via the uncinate fasciculus (Catani et al., 2002) is not involved in modular representations but rather in executive control functions that might impact on semantic processing. The roles of such connections and tracts in semantics remain unclear given contradicting findings. Most imaging studies in healthy adults, using various tasks tapping semantics and concomitantly also other capacities such as executive functioning and lexical competency, have suggested a semantic function of the so-called 'ventral stream' tracts including the ILF and the uncinate fasciculus (Hickok and Poeppel, 2004; Parker et al., 2005; Saur et al., 2008, 2010; Friederici and Gierhan, 2013). On the other side, intraoperative brain stimulation studies, directly mapping function and structure, have not confirmed the semantic involvement of these tracts (e.g., Mandonnet et al., 2007; Duffau, 2008).

The aim of the present study was to investigate the white matter network of semantics, presumably linking the ATL and remote cortices implementing cognitive modules. It furthermore aimed at clarifying the semantic role of the ILF and the uncinate fasciculus. To this end the study combined anatomical MRI (T1-weightes images), diffusion MRI and semantic assessments of verbal and non-verbal/visual stimuli in a large cohort of SD patients and healthy controls. We more specifically explored with probabilistic fiber tracking in healthy controls whether the ATL are connected to three regions implementing cognitive modules: the FFA 
(right hemisphere), the VWFA (left hemisphere) and lexical-related posterior parts of the left superior/middle temporal cortices. We then studied whether these anatomical connections are altered in SD, and whether they genuinely subtend semantics by conducting correlation analyses between tract parameters and scores of semantic tasks assessing verbal (words) and non-verbal object/face stimuli. Finally, we evaluated via correlation analyses the putative semantic roles of the ILF and the uncinate fasciculus. Post-hoc correlations were also conducted for the semantic tasks and control tracts, as well as for the tracts of interest and a task not involving semantic processing. One should note that this study focuses on module-hub connections that convey modular information, presumably automatically, to the semantic hub system to yield activation of concepts. It does not explore concept formation which occurs during the time course of human development or controlled semantic access which requires a more 'executive' component searching and retrieving information in the semantic system and which is referred to as 'semantic control' implemented mainly by prefrontal cortices (Labon Ralph et al., 2017).

\section{Methods}

\section{Participants}

Twenty-eight SD patients and 19 healthy controls were recruited from a French multicenter investigation on primary progressive aphasias ("PHRC-CAPP") including the SD variant. Diagnosis was established by expert neurologists following the international diagnostic criteria of Gorno-Tempini et al., (2011): progressive alteration of single-word comprehension, word finding and object knowledge, without agrammatism or motor speech disorders. All SD patients also responded to the imaging-supported criteria for SD diagnosis showing atrophy and hypometabolism of the ATL on MRI and FDG-PET (Gorno-Tempini et al., 2011). They were at an early stage of the disease as reflected by aphasia severity scores $\geq 3$ (normal $=5$ ) in the 
Boston Diagnostic Aphasia Examination (Mazaux and Orgogozo, 1982). Patients did not present with any neurological/psychiatric disease other than SD. The 19 healthy controls were matched with the patients for age, handedness, level of education and sex. All participants were native French speakers. The local ethics committee approved the study and informed written consent was obtained from the participants. Demographical information is summarized in Table 1.

Table 1 Demographical data of SD patients and healthy controls (means +/- standard deviations)

\begin{tabular}{lcc}
\hline & SD patients & Healthy controls \\
\hline Number of subjects & 28 & 19 \\
\hline Sex (women/men) & $13 / 15$ & $13 / 6$ \\
\hline Age (years) & $67.6+/-7.3$ & $64.9+/-6.2$ \\
\hline Handedness (right/left) & $27 / 1$ & $19 / 0$ \\
\hline Years of education & $13.2+/-5.3$ & $14.1+/-4.8$ \\
\hline Age at disease onset & $63.7+/-7.3$ & $/ / / / / / / / /$ \\
\hline Disease duration (years) & $1.2+/-1.04$ & $/ / / / / / / / /$ \\
\hline Symptom duration (years) & $3.9+/-2.5$ & $/ / / / / / / /$ \\
\hline
\end{tabular}




\section{Cognitive assessment}

\section{Standard cognitive/language assessment}

The general cognitive assessment included the Mini Mental State Examination (Folstein et al., 1975), the Mattis Dementia Rating Scale (Mattis, 1976), and the Frontal Assessment Battery (Dubois et al., 2000). The language assessment was based on the Boston Diagnostic Aphasia Evaluation (Mazaux \& Orgogozo, 1982) including an evaluation of the severity of aphasia, a sentence repetition task, and a single-word comprehension task. We also applied a picture naming test (DO80; Deloche et al., 1997), and a verbal fluency test comprising letter and category fluency (Cardebat et al., 1990).

The Raven's Progressive Matrices, which taps high level processes of analogical/rule reasoning without involving semantic representations/processes (Raven et al., 1998) was used as a control task for supplementary post-hoc analyses.

\section{Semantic tasks}

Semantic performance was assessed by three tasks tapping both the processing of verbal (words) and non-verbal stimuli (visual objects, famous faces): 1) A familiarity judgment and semantic matching task manipulating famous and non-famous people in a verbal version (names) and a non-verbal/visual version (black and white photographs) (SemPer battery; Laisney et al., 2009). The task was subdivided into three parts: a) 'familiarity judgment', b) matching with the professional domain, c) matching with subcategories of the professional domain. 2) A semantic matching task including a verbal version (written words) and a nonverbal/visual version (line drawings) (Pyramids and Palm Trees Test; Howard et al., 1992). 3) A second semantic matching task with verbal stimuli (written words) and of non-verbal/visual stimuli (line drawings) (BECS-GRECO; Merck et al., 2011). This task also included a supplementary condition of semantic matching in the non-verbal modality, referred to as 
‘identity matching', manipulating semantic/functional 'identity' and physical but non-semantic similarity. One should keep in mind that the use of picture/photograph stimuli to assess nonverbal aspects of semantics could induce a potential bias given that inner verbalization of such stimuli is possible, which is a near-universal and inevitable limitation of experimental tasks using visual stimuli to tap non-verbal processes.

In each participant, we identified a composite semantic score for both verbal and non-verbal modalities by calculating mean values derived from the percentage of correct responses of the three tasks and their respective verbal or non-verbal modalities. The three tasks are summarized in Table 2.

Table 2 Semantic tasks tapping verbal and non-verbal/visual modalities

\begin{tabular}{|c|c|}
\hline Verbal modality & Non-verbal modality \\
\hline \multicolumn{2}{|c|}{ SemPer battery } \\
\hline Familiarity judgment: names & Familiarity judgment: faces \\
\hline $\begin{array}{l}\text { Semantic matching } \\
\text { names - professional domain }\end{array}$ & $\begin{array}{l}\text { Semantic matching } \\
\text { faces - professional domain }\end{array}$ \\
\hline $\begin{array}{c}\text { Semantic matching } \\
\text { names }- \text { subcategories of professional domain }\end{array}$ & $\begin{array}{c}\text { Semantic matching } \\
\text { faces }- \text { subcategories of professional domain }\end{array}$ \\
\hline \multicolumn{2}{|c|}{ Pyramids and Palm Trees Test } \\
\hline Semantic matching: words & Semantic matching: pictures \\
\hline \multicolumn{2}{|c|}{ BECS-GRECO } \\
\hline Semantic matching: words & Semantic matching: pictures \\
\hline $\mid / / / / / I / /$ & 'Identity' matching \\
\hline
\end{tabular}




\section{Imaging}

MRI acquisitions and cognitive assessments were conducted within a one-month interval for all participants. All imaging centers involved in our investigation belong to the national network of the 'Centre d'Acquisition et de Traitement d'Images' (CATI) (http://cati-neuroimaging.com/) (Operto et al., 2016). MRI sequences were harmonized by the CATI in order to minimize differences between imaging centers. The CATI performs onsite visits for the setup of imaging protocols and regular follow-up. This rigorous quality and harmonizing procedure provided the rationale for not conducting statistical corrections for the use of different scanners.

Participants underwent whole-brain MRI scanning on 3-Tesla scanners at seven acquisition sites across France. Diffusion-weighted images (DWI) were acquired using an echo-planar imaging sequence $(\mathrm{EPI})\left(128 \times 128\right.$ acquired matrix, voxel size: $\left.2.0 \times 2.0 \times 2.0 \mathrm{~mm}^{3}\right)$. Seventy separate images were extracted from each DWI scan: ten T2-weighted images with no dedicated diffusion sensitization (b0 images) and sixty diffusion-weighted images ( $\left.b=1500 \mathrm{~s} / \mathrm{mm}^{2}\right)$. A fieldmap image was acquired to correct for geometrical distortions induced by the EPI sequence. Additionally, T1-weighted images were acquired with a 3D gradient echo sequence $\left(240 \times 256\right.$ acquired matrix; voxel size $=1.0 \times 1.0 \times 1.0 \mathrm{~mm}^{3}($ slice thickness $=1 \mathrm{~mm}) ;$ inversion time $=900 \mathrm{~ms}$; repetition time $2,300 \mathrm{~ms}$, echo time $=2.98 \mathrm{~ms}$; flip angle $\left.=9^{\circ}\right)$.

\section{Cortical ATL atrophy}

T1-weighted images were processed using the t1-freesurfer pipeline of the Clinica platform (http://www.clinica.run) which is a wrapper of different tools of the FreeSurfer image analysis software (http://surfer.nmr.mgh.harvard.edu) (Fischl, 2012). The processing pipeline included non-uniformity and intensity correction, skull stripping, grey/white matter segmentation, reconstruction of the cortical surface, cortical thickness estimation and spatial normalization onto the FreeSurfer surface template (FsAverage). After segmentation, all datasets were 
checked visually for segmentation errors (errors of GM/WM and GM/CSF boundaries). The SD group was compared to the healthy controls applying a point-wise, vertex-to-vertex model based on the Matlab SurfStat toolbox (http://www.math.mcgill.ca/keith/surfstat/) to analyze cortical thickness. The data were smoothed using a Gaussian kernel with a full width at half maximum (FWHM) set to $20 \mathrm{~mm}$.

\section{Identification of cortical regions of interest for fiber tracking}

We manually delineated the ATL and the cortical regions implementing modular representations (FFA, VWFA, lexical-related posterior parts of the left superior/middle temporal cortices). All cortical regions of interest were drawn on the FsAverage template using the FreeSurfer toolbox Tksurfer (https://surfer.nmr.mgh.harvard.edu/fswiki/TkSurfer) and then mapped onto each individual subject. The regions were defined according to the results of the functional neuroimaging literature. For all regions, their spatial extent was slightly enlarged compared to the results of group-wise functional neuroimaging imaging studies in order to account for inter-individual differences. We first delineated the ATL following the metaanalysis study of Rice et al. (2015). This region encompassed the most anterior regions of the temporal lobe, and anterior portions of the superior temporal gyrus, the middle temporal gyrus, the inferior temporal gyrus, the fusiform gyrus and the parahippocampal gyrus. We then identified the FFA and the VWFA as the right and left mid-posterior portion of the fusiform gyrus, respectively, based on previous functional MRI studies (Kanwisher et al., 1997; Cohen et al., 2000). The lexical-related temporal area was defined as a region encompassing the posterior parts of the superior temporal and middle temporal gyri in the left hemisphere according to previous imaging results (Kotz et al., 2002; Graves et al., 2008; Race and Hillis, 2015). The identified cortical regions are illustrated in Figure 1. 
These regions of interest were then mapped onto each individual subject. To that purpose, T1weighted images were processed using FreeSurfer image analysis software (stable version 5.3; http://surfer.nmr.mgh.harvard.edu). The processing pipeline included non-uniformity and intensity correction, skull stripping, grey/white matter segmentation, reconstruction of the cortical surface and segmentation of cortical structures. After segmentation, all datasets were checked visually for segmentation errors (errors of GM/WM and GM/CSF boundaries).

Finally, for each participant, the mean cortical thickness was computed within each of the identified cortical regions.

\section{Fiber tracking}

First, we aligned for each subject all raw DWI volumes to the average b0 image (DWI volume with no diffusion sensitization) with 6 degrees of freedom to correct for head motion, and the diffusion weighted directions were appropriately updated as recommended by Leemans and Jones (2009). A registration with 12 degrees of freedom was used to correct for eddy current distortions. These registrations were done using the FSL flirt tool (www.fmrib.ox.ac.uk/fsl). To correct for EPI-induced susceptibility artifacts, the fieldmap image was used as proposed by (Jezzard and Balaban, 1995) with the FSL prelude/fugue tools. Finally, the DWIs were corrected for non-uniform intensity using ANTs N4 bias correction algorithm (Tustison and Avants, 2013). A single multiplicative bias field from the averaged b0 image was estimated, as suggested by Jeurissen et al. (2014). The DWI dataset was up-sampled at $1 \mathrm{~mm}$ in order to improve the registration between the T1-weighted image and the DWI. A diffusion tensor model was fitted at each voxel to calculate Fractional Anisotropy (FA) and Mean Diffusivity (MD) maps.

We studied several white matter tracks connected to the ATL. The ILF and the uncinate fasciculus were explored in both hemispheres. Language-related tracts connecting the ATL to 
the VWFA, and the ATL to the lexical area in posterior parts of the left superior/middle temporal cortex were explored in the left hemisphere. Tracts connecting the ATL to the FFA were explored in the right hemisphere.

The ILF and the uncinate fasciculus were defined using the $J H U$ white-matter tractography atlas (Mori et al., 2005). Each tract of the $J H U$ atlas is defined as the probabilistic average of 28 normal subjects. Several thresholds of these probabilistic tracts are proposed $(0 \%, 25 \%$, $50 \%$ ). After visual inspection, the $25 \%$ threshold was selected which was neither too noisy (compared to the $0 \%$ threshold) nor too selective (compared to the 50\% threshold). For each subject, the FA map of the subject was registered onto the FA map of the $J H U$ atlas template with the ANTs SyN algorithm (Avants et al., 2008). Then, the estimated non-linear deformation was applied to the MD maps so that both the FA and MD maps of each subject were put into correspondence with the atlas.

Tracts connecting the ATL to module-implementing cortices (FFA, VWFA, lexical-related regions of the posterior temporal cortex) were identified by building a customized atlas of these tracts using fiber tracking. As fiber tracking might not be reliable in patients with severe atrophy we performed tractography in healthy controls in order to build the customized atlas, which was subsequently mapped onto each SD patient. Fiber tracking was performed as follows with the MRtrix software package (Brain Research Institute, Melbourne, Australia) (Tournier et al., 2012) (http://www.mrtrix.org). First, the fiber orientation distribution (FOD) was computed at highly anisotropic voxels $(\mathrm{FA}>0.7)$ to determine the response function, which was used for constrained spherical deconvolution to accurately estimate the FOD (Tournier et al., 2007). Then, fibers were generated with a probabilistic tracking algorithm that samples FOD at each step (Behrens et al., 2003). The algorithm generated 1.000.000 fibers of minimum length 20 $\mathrm{mm}$. Default tracking parameters were used (step size of $0.5 \mathrm{~mm}$, minimum radius of curvature of $1 \mathrm{~mm}$ and FOD cutoff of 0.1 ). All voxels in the 1-mm dilated white matter mask (obtained 
from the registered T1 image) were used as seeds. For each control subject, fibers passing through both the ATL and the FFA were selected thus forming the 'ATL-FFA tract'. Similarly, fibers passing through both the ATL and the VWFA formed the 'ATL-VWFA tract'. Finally, fibers passing through both the ATL and the lexical-related posterior parts of the left superior/middle temporal cortices ('Lexicon Area') formed the 'ATL-LA tract'. A trackdensity image (see Calamante et al., 2010) representing the density of fibers was generated for each tract. In order to normalize the track-density images onto the $J H U$ atlas, the non-linear deformation described in the previous paragraph was applied to the track-density images. The normalized track-density images of all healthy controls were averaged, empirically thresholded at $10 \%$ and binarized to obtain the final white matter tract masks. Finally, for each participant, the average FA and MD value was computed within each of the studied tracts.

As a supplementary post-hoc analysis, in addition to the above-mentioned tracts we also analyzed two control tracts to check for the predicted semantic roles of the three module-hub tracts: i) the cortico-spinal tract which, being a motor related structure, should not have any role in semantic processing, and ii) the right homologue of the 'ATL-LA tract' which should not have any role regarding the processing of the verbal modality of semantics. The cortico-spinal tract was defined with the same procedure used for the ILF and the UNC, based on the $J H U$ white-matter tractography atlas (Mori et al., 2005). The tracking of the right homologue of the 'ATL-LA tract' was based on the same procedure used for the left hemisphere 'ATL-LA tract', except that we selected fibers linking the right ATL to the cortical region that is the symmetric to the left hemisphere 'Lexicon Area' (posterior parts of the superior/middle temporal cortices).

\section{Statistical analysis}

We used non-parametric tests given that semantic task scores and tract measures were not normally distributed. Regarding semantic performance, we tested the effects of 'group' (SD patients, healthy controls) and 'task modality' (verbal, non-verbal) using a two-way rank-based 
analysis of linear models according to the algorithm proposed by Hocking (1985) and implemented in 'R' by Klokeand McKean (2012). Posthoc, we compared verbal and non-verbal task scores of SD patients and healthy controls using one sided two-sample Wilcoxon rank sum tests. Within each group (SD, controls) we also compared verbal and non-verbal performance using paired Wilcoxon signed rank. Regarding imaging data, we assessed differences in tract integrity (FA, MD) between SD patients and healthy controls using one sided two-sampled Wilcoxon rank sum tests. The relationship between semantic scores (verbal and non-verbal) and tract parameters was analyzed with Spearman's rank correlations. The correlation analyses were controlled for the effect of age and educational level. Correction for multiple comparisons was performed using the Bonferroni procedure, counting the number of comparison within each type of variable/test (group differences for semantic scores $(n=4)$, group differences for tract parameters $(n=16)$, correlations between tract parameters and semantic scores $(n=14))$. Supplementary analyses (control tracts, control task, mean cortical thickness, partial correlations) were conducted as follows. As done in the primary analyses, we assessed differences in tract parameters (FA, MD) for the control tracts (left and right corticospinal tract, right homologue of the 'ATL-LA' tract) between SD patients and healthy controls using one sided two-sampled Wilcoxon rank sum tests. To ensure that the resulting p-values were comparable to those of the primary analyses involving the tracts of interest, we applied the same level of correction for multiple comparisons $(n=16)$. As in the primary analyses, the relationships between semantic scores and the parameters of control tracts as well as between the control task (Raven's progressive matrices) and the different tract parameters was analyzed with Spearman's rank correlations. Again, we applied the same level of correction for multiple comparisons as in the primary analyses $(n=14)$. The same rationale was applied for correlations between mean cortical thickness of identified regions and semantic scores (verbal and nonverbal). Moreover, principal component analysis between the different measures from MRI 
(mean cortical thickness, MD) and semantic scores (verbal, non-verbal) was performed. In order to understand the implication of gray matter loss for the relation between semantic scores (verbal, non-verbal) and white matter tracts, Spearman's rank partial correlations were conducted. In all analyses, the statistical significance threshold was set to $p<0.05$. Statistical analyses were conducted using $R$ (version 3.1.1; R Core Team 2013).

\section{Results}

\section{Standard cognitive/langue tests}

Patients had a typical profile of SD showing impaired single-word comprehension, letter fluency, category fluency and picture naming contrasting with largely spared sentence repetition. Performance of SD patients and healthy controls are illustrated in Table 3.

Table 3 Results in the standard cognitive/language assessment of SD patients and healthy controls (means \pm standard deviations)

\begin{tabular}{|l|l|l|l|}
\hline & SD patients & Healthy controls & Normal threshold \\
\hline General cognitive tests & $24.96 \pm 2.7$ & $27.56 \pm 1.8$ & $\geq 27$ \\
\hline MMSE &
\end{tabular}




\begin{tabular}{|c|c|c|c|}
\hline FAB & $14.54 \pm 1.8$ & $17.11 \pm 0.8$ & $\geq 16$ \\
\hline MDRS & $118.75 \pm 11.9$ & $140.00 \pm 3.9$ & $\geq 137$ \\
\hline \multicolumn{4}{|l|}{ Language tests } \\
\hline Severity of aphasia (BDAE) & $3.89 \pm 0.57$ & $5 \pm 0$ & $\geq 4$ \\
\hline Single-word comprehension (BDAE) & $61.32 \pm 8.3$ & $71.63 \pm 0.6$ & $\geq 68$ \\
\hline Sentence repetition (BDAE) & $14.96 \pm 1.5$ & $15.84 \pm 0.7$ & $\geq 14$ \\
\hline DO80 & $43.51 \pm 20$ & $79.61 \pm 0.6$ & $\geq 75$ \\
\hline DO80 - semantic paraphasias & $9.11 \pm 6.4$ & $0.33 \pm 0.5$ & $\mid / / / / / / /$ \\
\hline Letter fluency (“P”/ 2 min) & $11.57 \pm 5.9$ & $25.31 \pm 7.1$ & $\geq 15$ \\
\hline Category fluency ("fruits" / 2 min) & $8.14 \pm 4.88$ & $21.36 \pm 5.6$ & $\geq 15$ \\
\hline
\end{tabular}

MMSE $=$ Mini-Mental State Examination, $\mathrm{FAB}=$ Frontal Assessment Battery, MDRS $=$ Mattis Dementia Rating Scale, BDAE = Boston Diagnostic Aphasia Evaluation, DO80 = picture naming test.

\section{Semantic tasks}

There was a significant effect of 'group' $(\mathrm{F}=171.4, \mathrm{p}<0.001)$ but not of 'task modality' $(\mathrm{F}=$ $0.02, \mathrm{p}=0.90)$. The interaction between 'group' and 'task modality' was not significant $(\mathrm{F}=$ $0.19, \mathrm{p}=0.66)$. Post-hoc tests with one sided two-sample Wilcoxon rank sum tests showed that SD patients performed poorer than healthy controls in both the verbal modality (patients: $69.36 \%$ correct \pm 13.12 , controls: $97.6 \%$ correct $\pm 2.13 ; \mathrm{W}=0, \mathrm{p}<0.001)$ and the non-verbal modality (patients: $72.81 \%$ correct \pm 10.78 , controls: $96.55 \%$ correct $\pm 3.39 ; \mathrm{W}=6, \mathrm{p}<0.001$ ) Furthermore, there was no difference between scores of the verbal and non-verbal modality in SD patients $(\mathrm{V}=138, \mathrm{p}=0.56)$ or in healthy controls $(\mathrm{V}=118, \mathrm{p}=0.65)$. Results are illustrated in Figure 2. 
Performance in the control task (Raven's Progressive Matrices) was similar in SD patients and healthy controls (patients $85.03 \%$ correct \pm 4.3 , healthy controls 88.13 correct $\pm 3.4 ; \mathrm{W}=232$, $\mathrm{p}=0.24)$

\section{Imaging}

\section{ATL atrophy and fiber tracking in healthy controls}

Cortical thickness of the left and right ATL was reduced in SD patients, slightly predominating in the left ATL and caudally extending beyond the left ATL region (see Figure 3). Individual findings of ATL atrophy also showed that 7 SD patients had predominant right ATL atrophy and that the remaining 21 patients had predominant left ATL atrophy.

Fiber tracking identified a dense set of fibers connecting the ATL to the FFA and the VWFA, following the ventral part of the right and left temporal lobe, respectively, and to the lexicalrelated left posterior/superior temporal regions ('Lexicon Area') following more dorsal parts of the left temporal lobe. We will refer to these module-hub fiber bundles as the 'ATL-FFA tract', the 'ATL-VWFA tract', and the 'ATL-LA tract'. The tracts are illustrated in Figure 4. We also identified the right homologue of the 'ATL-LA tract'.

We then analyzed the percentages of overlap between the different tracts by calculating the intersection between them (pair-wise analyses) and dividing the number of intersection voxels by the total number of voxels of each pair of tracts. The analyses showed that the lefthemisphere 'ATL-LA tract' and 'ATL-VWFA tract' demonstrate no overlap, and that there was only poor overlap between these two tracts and the left ILF (respectively, 18.6\% and 2.7\%). Likewise, the right-hemisphere 'ATL-FFA tract' has only poor overlap with the right ILF $(16.0 \%)$. Finally, the uncinate fasciculus had no overlap with any of the other tracts. 


\section{Comparisons of tract integrity between SD patients and healthy controls}

There were significant tract alterations in SD patients for MD measures regarding the uncinate fasciculus (bilaterally), the ILF (bilaterally), the 'ATL-LA tract', the 'ATL-VWFA tract' and the 'ATL-FFA tract' (Table 4). For FA measures we found significant differences only for the uncinate fasciculus (bilaterally) and the 'ATL-VWFA tract'. MD therefore appeared to be more sensitive than FA to white matter changes, which has already been reported in previous tractography studies on SD (Agosta et al., 2010). We therefore used MD measures for the subsequent correlation analyses with semantic scores. Regarding the two control tracts which were predicted not to be involved in semantics (cortico-spinal tract), and, more specifically not to contribute to verbal semantics (right homologue of the 'ATL-LA tract'), we found that the former tract is not altered in our SD patients (bilaterally) and that the latter is altered for MD values. Results are summarized in Table 5.

In addition, we compared in SD patients FA and MD measures between the left and right hemisphere tracts which were bilaterally explored in the study (paired Wilcoxon signed rank tests). Alterations were more important in the left hemisphere compared to the right for the uncinate fasciculus $(V=50, p<0.001$ for $F A, V=339, p<0.01$ for MD) and the ILF (V = 10, $\mathrm{p}=<0.001$ for $\mathrm{FA} ; \mathrm{V}=328, \mathrm{p}<0.01$ for $\mathrm{MD}$ ). The same holds for 'ATL-LA tract' as compared to its right homologue $(\mathrm{V}=395, \mathrm{p}<0.001$ for $\mathrm{FA} ; \mathrm{V}=279, \mathrm{p}=0.085$ for MD) and the left hemisphere 'ATL- VWFA tract' compared to its right homologue 'ATL- FFA tract' (V=2, p $<0.001$ for $\mathrm{FA} ; \mathrm{V}=364, \mathrm{p}<0.001$ for $\mathrm{MD})$. 
Table 4 Comparisons of tract parameters (MD, FA) in SD patients and healthy controls (mean values \pm standard deviations). MD is expressed in $\mathrm{mm}^{2} / \mathrm{s}$. P-values are corrected for multiple comparisons (16 tests)

\begin{tabular}{|c|c|c|c|c|c|}
\hline & Parameter & Tract & $\begin{array}{c}\text { SD } \\
\text { patients }\end{array}$ & Controls & $\begin{array}{c}\text { Wilcoxon rank sum } \\
\text { test }\end{array}$ \\
\hline \multirow{8}{*}{$\begin{array}{c}\text { Left } \\
\text { hemisphere }\end{array}$} & \multirow{4}{*}{ FA } & $\begin{array}{l}\text { Uncinate } \\
\text { fasciculus }\end{array}$ & $\begin{array}{l}0.25 \pm \\
0.04\end{array}$ & $\begin{array}{l}0.33 \pm \\
0.04\end{array}$ & $\mathrm{~W}=61, \mathrm{p}<0.001$ \\
\hline & & ILF & $0.32 \pm 0.03$ & $0.33 \pm 0.04$ & $\mathrm{~W}=238, \mathrm{p}=1.000$ \\
\hline & & $\begin{array}{l}\text { ATL-VWFA } \\
\text { tract }\end{array}$ & $0.20 \pm 0.03$ & $0.22 \pm 0.02$ & $\mathrm{~W}=134, \mathrm{p}=0.029$ \\
\hline & & ATL-LA tract & $0.31 \pm 0.03$ & $0.34 \pm 0.03$ & $\mathrm{~W}=159, \mathrm{p}=0.150$ \\
\hline & \multirow{4}{*}{ MD } & $\begin{array}{l}\text { Uncinate } \\
\text { fasciculus }\end{array}$ & $1.20 \pm 0.21$ & $0.78 \pm 0.03$ & $\mathrm{~W}=531, \mathrm{p}<0.001$ \\
\hline & & ILF & $0.92 \pm 0.15$ & $0.73 \pm 0.03$ & $\mathrm{~W}=516, \mathrm{p}<0.001$ \\
\hline & & $\begin{array}{l}\text { ATL-VWFA } \\
\text { tract }\end{array}$ & $1.23 \pm 0.31$ & $0.75 \pm 0.04$ & $\mathrm{~W}=532, \mathrm{p}<0.001$ \\
\hline & & ATL-LA tract & $0.92 \pm 0.13$ & $0.74 \pm 0.03$ & $\mathrm{~W}=504, \mathrm{p}<0.001$ \\
\hline \multirow{6}{*}{$\begin{array}{c}\text { Right } \\
\text { hemisphere }\end{array}$} & \multirow{3}{*}{ FA } & $\begin{array}{l}\text { Uncinate } \\
\text { fasciculus }\end{array}$ & $0.31 \pm 0.06$ & $0.35 \pm 0.05$ & $\mathrm{~W}=134, \mathrm{p}=0.029$ \\
\hline & & ILF & $0.37 \pm 0.05$ & $0.37 \pm 0.03$ & $\mathrm{~W}=249, \mathrm{p}=1.000$ \\
\hline & & ATL-FFA tract & $0.28 \pm 0.05$ & $0.28 \pm 0.03$ & $\mathrm{~W}=264, \mathrm{p}=1.000$ \\
\hline & \multirow{3}{*}{ MD } & $\begin{array}{l}\text { Uncinate } \\
\text { fasciculus }\end{array}$ & $0.97 \pm 0.30$ & $0.73 \pm 0.30$ & $\mathrm{~W}=457, \mathrm{p}<0.001$ \\
\hline & & ILF & $0.80 \pm 0.13$ & $0.69 \pm 0.13$ & $\mathrm{~W}=417, \mathrm{p}=0.006$ \\
\hline & & ATL-FFA tract & $0.81 \pm 0.77$ & $0.73 \pm 0.77$ & $\mathrm{~W}=461, \mathrm{p}<0.001$ \\
\hline
\end{tabular}


Table 5 Supplementary analysis. Comparisons of control tract parameters (MD, FA) in SD patients and healthy controls (mean values \pm standard deviations). MD is expressed in $\mathrm{mm}^{2} / \mathrm{s}$. In order to ensure that $\mathrm{p}$-values are comparable to those reported in Table 4, they were corrected for the same number of multiple comparisons (16 tests).

\begin{tabular}{|c|c|c|c|c|c|}
\hline & Parameter & Tract & $\begin{array}{c}\text { SD } \\
\text { patients }\end{array}$ & Controls & $\begin{array}{c}\text { Wicoxon rank } \\
\text { sum test }\end{array}$ \\
\hline \multirow{2}{*}{$\begin{array}{c}\text { Left } \\
\text { hemisphere }\end{array}$} & $\mathbf{F A}$ & $\begin{array}{l}\text { Control tract: } \\
\text { Cortico-spinal } \\
\text { tract }\end{array}$ & $\begin{array}{c}0.51 \pm \\
0.06\end{array}$ & $\begin{array}{c}0.50 \pm \\
0.03\end{array}$ & $\begin{array}{l}W=234 \\
p=1\end{array}$ \\
\hline & MD & $\begin{array}{l}\text { Control tract: } \\
\text { Cortico-spinal } \\
\text { tract }\end{array}$ & $\begin{array}{c}0.71 \pm \\
0.13\end{array}$ & $\begin{array}{c}0.67 \pm \\
0.04\end{array}$ & $\begin{array}{l}W=344 \\
p=0.745\end{array}$ \\
\hline \multirow{4}{*}{$\begin{array}{c}\text { Right } \\
\text { hemisphere }\end{array}$} & \multirow{2}{*}{$\mathbf{F A}$} & $\begin{array}{l}\text { Control tract: } \\
\text { Cortico-spinal } \\
\text { tract }\end{array}$ & $\begin{array}{c}0.52 \pm \\
0.06\end{array}$ & $\begin{array}{c}0.52 \pm \\
0.04\end{array}$ & $\begin{array}{l}W=239 \\
p=1\end{array}$ \\
\hline & & $\begin{array}{l}\text { Control tract: } \\
\text { right homologue } \\
\text { of ATL-LA tract }\end{array}$ & $\begin{array}{c}0.30 \pm \\
0.04\end{array}$ & $\begin{array}{c}0.33 \pm \\
0.03\end{array}$ & $\begin{array}{l}W=250 \\
p=1\end{array}$ \\
\hline & \multirow{2}{*}{ MD } & $\begin{array}{l}\text { Control tract: } \\
\text { Cortico-spinal } \\
\text { tract }\end{array}$ & $\begin{array}{c}0.69 \pm \\
0.15\end{array}$ & $\begin{array}{c}0.64 \pm \\
0.54\end{array}$ & $\begin{array}{l}W=340 \\
p=0.893\end{array}$ \\
\hline & & $\begin{array}{l}\text { Control tract: } \\
\text { right homologue } \\
\text { of ATL-LA tract }\end{array}$ & $\begin{array}{c}0.90 \pm \\
0.14\end{array}$ & $\begin{array}{c}0.72 \pm \\
0.03\end{array}$ & $\begin{array}{l}\mathrm{W}=443 \\
\mathrm{p}<0.001\end{array}$ \\
\hline
\end{tabular}




\section{Relationship between tract integrity and semantic performance}

The results of the correlation analyses using composite semantic scores for the verbal and nonverbal task modalities and tract parameters (MD) are summarized in Table 6. Non-verbal semantic scores were significantly correlated with MD measures of all explored tracts in the right hemisphere: the uncinate fasciculus, the ILF and the 'ATL-FFA tract'. Verbal semantic scores were significantly correlated to MD measures of two left hemisphere tracts: the 'ATLVWFA tract' and the 'ATL-LA tract'. No correlations were found between non-verbal semantic scores and the left hemisphere tracts or verbal semantic scores and the right hemisphere tracts. Results of supplementary analyses (control tracts and control task) are presented in Table 7. Regarding the two control tracts, there were significant correlations between the right corticospinal tract integrity and non-verbal semantic scores and between the right homologue of the 'ATL-LA tract' and non-verbal semantic scores. Finally, regarding the control task (Raven's Progressive Matrices), there were no significant correlations between task scores and the 'ATLLA tract', the 'ATL-VWFA tract', the 'ATL-FFA tract', the ILF or the uncinate fasciculus. 
Table 6 Results of correlation analyses using tract measures of MD and composite semantic verbal and non-verbal/visual scores, as well as control task scores. P-values are corrected for multiple comparisons (14 tests)

\begin{tabular}{|c|c|c|c|c|c|}
\hline \multirow{2}{*}{$\begin{array}{l}\text { Semantic } \\
\text { modality }\end{array}$} & \multirow{2}{*}{ Tracts } & \multicolumn{2}{|c|}{$\begin{array}{c}\text { Left } \\
\text { hemisphere }\end{array}$} & \multicolumn{2}{|c|}{ Right hemisphere } \\
\hline & & Rho & p-value & Rho & p-value \\
\hline \multirow{5}{*}{ Verbal } & Uncinate fasciculus & -0.11 & 1 & -0.45 & 0.299 \\
\hline & ILF & -0.54 & 0.068 & -0.40 & 0.633 \\
\hline & ATL-VWFA tract & -0.64 & 0.007 & ////// & $/ / / / / /$ \\
\hline & ATL-FFA tract & $/ / / / /$ & $/ / / / / /$ & -0.55 & 0.050 \\
\hline & ATL-LA tract & -0.58 & 0.029 & ////// & $/ / / / / /$ \\
\hline \multirow{5}{*}{ Non-verbal } & Uncinate fasciculus & 0.23 & 1 & -0.67 & 0.002 \\
\hline & ILF & -0.18 & 1 & -0.61 & 0.012 \\
\hline & ATL-VWFA tract & -0.24 & 1 & $/ / / / / /$ & $/ / / / / /$ \\
\hline & ATL-FFA tract & ////// & $/ / / / / /$ & -0.75 & $<0.001$ \\
\hline & ATL-LA tract & -0.30 & 1 & $/ / / / / /$ & $/ / / / / /$ \\
\hline
\end{tabular}


Table 7 Supplementary analysis. Results of correlation analyses between control tract measures of MD and composite semantic verbal and non-verbal/visual scores and between all studied tracts and the control task (Raven's progressive matrices) scores. In order to ensure that pvalues are comparable to those reported in Table 4, they were corrected for the same number of multiple comparisons (14 tests).

\begin{tabular}{|c|c|c|c|c|c|}
\hline \multirow{2}{*}{$\begin{array}{l}\text { Semantic } \\
\text { modality }\end{array}$} & \multirow{2}{*}{ Tract } & \multicolumn{2}{|c|}{ Left hemisphere } & \multicolumn{2}{|c|}{ Right hemisphere } \\
\hline & & Rho & p-value & Rho & p-value \\
\hline \multirow[b]{2}{*}{ Verbal } & $\begin{array}{l}\text { Control tract: Cortico- } \\
\text { spinal tract }\end{array}$ & -0.40 & 0.593 & -0.54 & 0.059 \\
\hline & $\begin{array}{l}\text { Control tract: right } \\
\text { homologue of ATL-LA } \\
\text { tract }\end{array}$ & $/ / /$ & $/ / /$ & -0.52 & 0.075 \\
\hline \multirow{2}{*}{$\begin{array}{l}\text { Non- } \\
\text { verbal }\end{array}$} & $\begin{array}{l}\text { Control tract: Cortico- } \\
\text { spinal tract }\end{array}$ & -0.34 & 1 & -0.55 & 0.049 \\
\hline & $\begin{array}{l}\text { Control tract: right } \\
\text { homologue of ATL-LA } \\
\text { tract }\end{array}$ & $/ / /$ & $/ / /$ & -0.78 & $<0.001$ \\
\hline \multirow{7}{*}{$\begin{array}{c}\text { Control } \\
\text { task } \\
\text { (Raven's } \\
\text { Matrices) }\end{array}$} & Uncinate fasciculus & 0.07 & 1 & -0.41 & 0.516 \\
\hline & ILF & -0.12 & 1 & -0.38 & 0.724 \\
\hline & ATL-VWFA tract & -0.10 & 1 & $/ / /$ & $/ / /$ \\
\hline & ATL-FFA tract & $/ / /$ & $/ / /$ & -0.41 & 0.475 \\
\hline & ATL-LA tract & -0.21 & 1 & $/ / /$ & $/ / /$ \\
\hline & $\begin{array}{l}\text { Control tract: right } \\
\text { homologue of ATL-LA } \\
\text { tract }\end{array}$ & $/ / /$ & $/ / /$ & -0.44 & 0.319 \\
\hline & $\begin{array}{l}\text { Control tract: Cortico- } \\
\text { spinal tract }\end{array}$ & -0.20 & 1 & -0.15 & 1 \\
\hline
\end{tabular}




\section{Relationship between gray matter loss and semantic performance}

The results of the correlation analyses using composite semantic scores for the verbal and nonverbal task modalities and mean cortical thickness are summarized in Table 8. Non-verbal semantic scores were significantly correlated with one region in the right hemisphere: the right ATL. No correlations were found between non-verbal semantic scores and regions in the left hemisphere or verbal semantic scores and any of the identified cortical regions.

Results of the principal component analysis for MRI features (mean cortical thickness, MD) and semantic scores (verbal, non-verbal) are shown in the Supplementary Figure. The first principal component (horizontal axis), representing 39\% of the total variance, seems to represent the level of neurodegeneration. Indeed, it opposes mean cortical thickness to white matter loss: the thinner the mean cortical thickness of a patient, the larger the mean diffusivity (and thus the larger the white matter loss). The second principal component (vertical axis), representing $29 \%$ of the total variance, seems to differentiate MRI features from the left and the right hemisphere as well as verbal and non-verbal scores. It should be noted that verbal scores are associated with left hemisphere MRI features whereas non-verbal scores are associated to right hemisphere MRI features. Together, these two axes seem to indicate an effect of lateralization associating, on the one hand, the impairment of non-verbal semantics with neurodegeneration of the studied gray and white matter in the right hemisphere, and, on the other hand, the impairment of verbal semantics with neurodegeneration of the gray and white matter in the left hemisphere. 
Table 8. Results of correlation analyses between mean cortical thickness and composite semantic verbal and non-verbal/visual scores. In order to ensure that p-values are comparable to those reported in Table 4, they were corrected for the same number of multiple comparisons (14 tests).

\begin{tabular}{|c|l|c|c|c|c|}
\hline \multirow{2}{*}{$\begin{array}{c}\text { Semantic } \\
\text { modality }\end{array}$} & \multicolumn{1}{|c|}{ Cortical region } & \multicolumn{2}{c|}{$\begin{array}{c}\text { Left } \\
\text { hemisphere }\end{array}$} & \multicolumn{2}{c|}{$\begin{array}{c}\text { Right } \\
\text { hemisphere }\end{array}$} \\
\cline { 2 - 7 } & & Rho & p-value & Rho & p-value \\
\hline \multirow{5}{*}{ Verbal } & Anterior Temporal Lobe (ATL) & 0.31 & 1 & 0.48 & 0.16 \\
\cline { 2 - 7 } & Visual Word Form Area & 0.23 & 1 & - & - \\
\cline { 2 - 7 } & Fusiform Face Area & - & - & 0.15 & 1 \\
\cline { 2 - 7 } & $\begin{array}{l}\text { Lexicon Area (LA) / } \\
\text { Right homologue of LA }\end{array}$ & 0.53 & 0.07 & 0.15 & 1 \\
\hline \multirow{5}{*}{ Non-verbal } & ATL & -0.06 & 1 & $\mathbf{0 . 7 4 4}$ & $<\mathbf{0 . 0 0 1}$ \\
\cline { 2 - 7 } & Visual Word Form Area & 0.12 & 1 & - & - \\
\cline { 2 - 7 } & Fusiform Face Area & - & - & 0.50 & 0.11 \\
\cline { 2 - 7 } & $\begin{array}{l}\text { LA / } \\
\text { Right homologue of LA }\end{array}$ & 0.15 & 1 & 0.54 & 0.051 \\
\hline
\end{tabular}

Implication of the gray matter loss of gray matter loss for the relation between semantic performance and white matter integrity

The results of the partial correlations analyses between composite semantic scores (verbal and non-verbal task modalities) and tract integrity, corrected for the mean cortical thickness of the region(s) connected to these tracts are summarized in Table 9. Verbal semantic scores remained significantly correlated with the 'ATL-VWFA' after inclusion of the ATL and/or the VWFA regions. Non-verbal semantic scores remained significantly correlated for two tracts in the right 
hemisphere: the 'ATL-FFA tract' corrected for the FFA region and the right homologue of 'ATL-LA tract' corrected for the right homologue of LA region. 
Table 9. Results of partial correlation analyses using tract measures of MD and composite semantic verbal and non-verbal/visual scores partialled out for cortical thickness measures. In order to ensure that p-values are comparable to those reported in Table 4, they were corrected for the same number of multiple comparisons (14 tests).

\begin{tabular}{|c|c|c|c|c|}
\hline \multirow{2}{*}{$\begin{array}{l}\text { Semantic } \\
\text { modality }\end{array}$} & \multirow{2}{*}{ Tracts } & \multirow{2}{*}{$\begin{array}{l}\text { Corrected } \\
\text { for }\end{array}$} & \multicolumn{2}{|c|}{ Left hemisphere } \\
\hline & & & Rho & p-value \\
\hline \multirow{8}{*}{ Verbal } & Uncinate fasciculus & ATL & 0.06 & 1 \\
\hline & ILF & ATL & -0.45 & 0.29 \\
\hline & \multirow{3}{*}{ ATL-VWFA tract } & ATL & -0.63 & 0.009 \\
\hline & & VWFA & -0.61 & 0.015 \\
\hline & & $\begin{array}{l}\text { ATL + } \\
\text { VWFA }\end{array}$ & -0.63 & 0.013 \\
\hline & \multirow{3}{*}{ ATL-LA tract } & ATL & -0.50 & 0.13 \\
\hline & & LA & -0.34 & 1 \\
\hline & & $\mathrm{ATL}+\mathrm{LA}$ & -0.35 & 1 \\
\hline & & & \multicolumn{2}{|c|}{ Right hemisphere } \\
\hline & & & Rho & p-value \\
\hline \multirow{8}{*}{$\begin{array}{l}\text { Non- } \\
\text { verbal }\end{array}$} & Uncinate fasciculus & ATL & -0.15 & 1 \\
\hline & ILF & ATL & -0.001 & 1 \\
\hline & \multirow{3}{*}{ ATL-FFA tract } & ATL & -0.24 & 1 \\
\hline & & FFA & -0.64 & 0.006 \\
\hline & & $\mathrm{ATL}+\mathrm{FFA}$ & -0.24 & 1 \\
\hline & \multirow{3}{*}{$\begin{array}{l}\text { Right homologue of } \\
\text { ATL-LA tract }\end{array}$} & ATL & -0.42 & 0.47 \\
\hline & & LA & -0.66 & 0.003 \\
\hline & & $\mathrm{ATL}+\mathrm{LA}$ & -0.43 & 0.52 \\
\hline
\end{tabular}




\section{Discussion}

The present investigation explored a large population of SD patients and healthy controls to identify the white matter network of semantics essential for conveying modular information from posterior cortices to the supra-modal hub in the ATL. We first delineated three welldocumented module-related cortices and performed probabilistic fiber tracking in healthy controls to identify the tracts connecting these cortical regions to the ATL. In a second step, we analyzed the alterations of these tracts in $\mathrm{SD}$, and conducted correlations analyses with tract parameters and semantic scores of verbal and non-verbal tasks to investigate the semantic roles of the different tracts. Finally, we evaluated the putative semantic functions of the ILF and the uncinate fasciculus which have been shown to project to the ATL.

Our results reveal the existence of several tracts projecting from cortical modules to the semantic ATL hub: the 'ATL-FFA tract', the 'ATL-VWFA tract', and the 'ATL-LA tract'. Our findings show that all tracts are microstructurally altered in SD, and that they subserve specific semantic functions related to the transfer of modular verbal information to the left ATL ('ATLVWFA tract', 'ATL-LA tract') and of modular non-verbal/visual information to the right ATL ('ATL-FFA tract'). Furthermore, the semantic roles of the ILF and the uncinate fasciculus appear to be restricted to non-verbal operations in the right hemisphere. These findings have implications at multiples levels: anatomically, they enrich the knowledge of the white matter network converging onto the ATL; neuro-cognitively, they elucidate major components of the semantic network conveying modular information to the supra-modal hub of semantics; finally, regarding $\mathrm{SD}$, they highlight wide-spread microstructural and functional damage to the semantic network extending beyond the ILF and the uncinate fasciculus.

\section{Anatomical connections between the ATL and remote cortices}

Since the creation of fiber tracking techniques numerous imaging studies have identified the major structural connections inter-linking grey matter regions, but most of these studies were 
anatomically-driven and did not build on cognitive/functional models. They have identified some tracts probably contributing to language or semantic processing including the ILF, the superior longitudinal fasciculus comprising the arcuate, the uncinate fasciculus and the inferior frontal-occipital fasciculus (Mandonnet et al., 2007; Duffau, 2008; Saur et al., 2008, 2010; Friederici and Gierhan, 2013). However, there is still a huge gap between the complexity and multitude of language/semantic processes and the relatively small number of explored tracts. Regarding semantics, the core computations are claimed to be implemented by the supra-modal ATL hub but structural connections between the ATL and remote module-implementing cortices have not been investigated. Only two ATL connections, the ILF and the uncinate fasciculus, have been highlighted, yet without considering whether they might connect the ATL to cortical input modules for semantics. The same holds for a less documented tract referred to as the middle longitudinal fasciculus (Makris et al., 2009, 2013, 2017).

We addressed these open issues in healthy adults by exploring ATL tracts specifically connecting cortices implementing modular representations, along with the two welldocumented ATL connections. One novelty of our tracking study was the identification of tracts connecting the ATL to three modular cortical regions which implement i) written word forms: the 'ATL-VWFA tract', ii) abstract lexical representations: the 'ATL-LA tract', and iii) face/object representations: the 'ATL-FFA tract'. Previous tracking studies did not provide evidence for these tracts probably because they did not explicitly search such connections given that they were hardly based on a cognitive module-driven approach. Furthermore, previous studies in healthy adults often attempted to replicate connectivity findings from animals/primates which do not have a human-like semantic processing system. There are, however, two exceptions suggesting connections between the ATL and module-implementing cortices. First, connections between the VWFA and the ATL have been identified by Bouhali et al. (2014), but this investigation did not check for their semantic role. Second, fiber tracking 
studies on the macaque monkey have identified the so-called middle longitudinal fasciculus (MLF) which might eventually overlap with our 'ATL-LA tract'. However, there are only few investigation in human adults confirming the existence of the MLF, and its involvement in semantics is still debated (Makris et al., 2009, 2013, 2017; Jouen et al., 2018). Furthermore, there is currently no published atlas for the MLF which makes it difficult to know whether this tract corresponds to, or partly overlaps with our 'ATL-LA tract'.

In summary, the present tracking findings in healthy adults enrich and extend the results of previous studies by providing evidence for the existence of an anatomical ATL network, connecting the ATL to specific remote cortices involved in cognitive modules that require subsequent semantic processing. However, the study of healthy controls could not clarify whether these module-hub tracts are genuinely involved in semantics given the lack of variability of semantic task scores (near-ceiling effects), which impeded the rational for conducting correlation analyses between tract parameters and semantic scores. Conversely, SD patients provide the opportunity to address this anatomo-functional issue.

\section{Semantic roles of ATL tracts}

A major issue was to check whether the 'ATL-FFA tract', the 'ATL-VWFA tract', and the 'ATL-LA tract' play a genuine role in semantic processing. Our correlation analyses with SD patients bear on this issue indicating that the three tracts are related to semantic processes, and that the left hemisphere 'ATL-VWFA tract' and 'ATL-LA tract' convey modular language information to the semantic hub whereas the right hemisphere 'ATL-FFA tract' transfers information of modular face and visual object representations. The specific semantic roles of these tracts are further strengthened by our results on a control task showing that scores of the Raven's Progressive Matrices, which taps high level processes of analogical/rule reasoning without involving semantic representations/processes, do not correlate with parameters of any 
of the three module-hub tracts. Furthermore, the right homologue of the 'ATL-LA tract', which was used as a control tract regarding the left hemisphere 'ATL-LA tract', does not correlate with scores of verbal semantics but only with those of non-verbal semantics. On the contrary, the right motor-related cortico-spinal tract, also used as a control tract, correlates with scores of non-verbal semantics. This is an unexpected result which might possibly suggest that patients with strong non-verbal semantic impairment have a more extensive right hemisphere damage, including the right cortical-spinal tract, although the tract was not significantly damaged regarding its $\mathrm{FA} / \mathrm{MD}$ parameters.

The observed specific connections between language and non-language/visual modules and the semantic system are consistent with cognitive models of language and object processing. In language, the majority of psycholinguistic models assume that the mental lexicon and the semantic system are directly linked (Caramazza, 1997; Levelt et al., 1999; Indefrey and Levelt, 2004), which allows for the extraction of meaning from word forms during language perception, and the access to semantically appropriate word forms during language production. These cognitive links between word forms and semantic representations are materialized by two tracts identified in our study: the 'ATL-LA tract' and the 'ATL-VWFA tract' connecting the ATL to posterior temporal cortices implementing the mental lexicon (e.g., Kotz et al., 2002; Graves et al., 2007, 2008; Race and Hillis, 2015) and to the mid-posterior region of the left fusiform gyrus implementing orthographical word forms (Cohen et al., 2002; McCandliss et al., 2003). A similar architecture holds for visual object recognition in cognitive models which state the existence of a repertory of visual object/face forms, referred to as the 'structural description system' (Lloyd-Jones and Humphreys, 1997; Gerlach et al., 1999). This modular repertory is assumed to be linked to the semantic system allowing for the extraction of object/face meanings. Such cognitively modeled links are, at least partly, embodied by the 'ATL-FFA tract' connecting the ATL to the mid-posterior region of the right fusiform gyrus implementing face 
and object representations (Kanwisher et al., 1997; Gerlach et al., 1999; Tarr and Gauthier, 2000; Xu, 2005). Our findings represent a first step revealing the existence of anatomofunctional connections linking modules to the semantic hub system and, more generally, providing anatomical evidence corroborating major cognitive models and investigations of the modular organization of cognition (Fodor, 1983; Caramazza and Miozzo 1997; Levelt, 1999; Indefrey and Levelt 2004; Pinker and Jackendoff, 2005; Tyler et al., 2005; Ullman, 2001). Based on our findings and on cognitive models accounting for modules, unlike connectionist models, we propose to consider an extension of the 'hub-and-spokes' model of semantics (Lambon Ralph et al., 2017) to a 'hub-module-spokes' model where spokes represent/process motor-sensory features as stipulated by the authors of the model. Such motor-sensory features that are complex, highly variable and near-unlimited in number, are concatenated into invariant and robust modular representations (verbal - lexical representations, and non-verbal - visual object representations), which are limited in number and which encode the limited number of verbal and non-verbal objects of the human environment. These modular representations might constitute the operational and exploitable basis for the central semantic hub-system that forms, activates and generalizes concepts, the number of which is also limited like real-world objects. The identified tracts materializing module-hub connections also enrich previous anatomical investigations which have explored multiple connections between the ATL and remote temporal, parietal and frontal regions, yet without assessing their specific roles in semantics (Binney et al., 2012; Pascual et al., 2015; Jouen et al., 2018). Furthermore, our results are in line with several authors suggesting that semantic processing is subserved by a ventral stream pathway including the ILF, the uncinate fasciculus (e.g., Saur et al., 2008, 2010) and the inferior fronto-occipital pathway (Duffau et al., 2005; Mandonnet et al., 2007; Duffau, 2008), while identifying the critical module-hub tracts within this ventral stream, and assigning specific semantic roles to them. 
These findings open an avenue for future research that need to replicate our findings in large settings of other patient populations using, in addition to correlation analyses, disruption of these tracts to further substantiate their causative role in semantics. Likewise functional connectivity studies based on fMRI resting-state might confirm and enrich the existence of the semantic roles of the 'ATL-FFA', the 'ATL-VWFA' and the 'ATL-LA tract'. Another open issue is the exploration of contralateral homologues of these tracts and of the three modulerelated cortices which were investigated in this study. Our results on control tracts showed that parameters of the right homologue of the 'ATL-LA tract' correlated with task scores of nonverbal semantics, which suggests that, in addition to the FFA, the right posterior/superior temporal cortex might have a role in the implementation of modular representations of visual objects, and that the right homologue of the 'ATL-LA tract' might convey modular information of visual object representations to the right ATL. Future research is required to further address these issues with the aim of improving our understanding of the anatomical organization of cognitive modules and of the semantic module-hub network. Additional research is also needed to explore whether the bilateral ATL hub represents a unified system dedicated to supra-modal semantics or whether there is a left versus right specialization or gradient. In particular, left versus right hemisphere module-hub tracts, such as those evidenced in the present study, might provide input for verbal versus non-verbal/visual semantic computations in the left and right ATL, respectively. Providing evidence for this assumption would strengthen the claim of several authors positing that verbal semantics is primarily processed in the left ATL whereas visual non-verbal semantics might be computed in the right ATL (Snowden et al., 2004; Gainotti, 2013; Mesulam et al., 2013).

Regarding the semantic functions of the ILF and the uncinate fasciculus our results suggest that their semantic contribution is restricted to their right hemisphere fiber bundles, and that their semantic roles in conveying non-verbal information to the ATL hub are less salient than the 
role of the 'ATL-FFA tract'. Accordingly, only the right hemisphere ILF and uncinate fasciculus were correlated with semantic performance restricted to non-verbal/visual task modalities. In the literature, the exact semantic roles of the ILF and the uncinate fasciculus are not fully elucidated because prior studies provided partially contradicting results regarding a potential semantic function of these tracts, or did not contrast verbal and non-verbal modalities (Mandonnet et al., 2007; Duffau, 2008; Friederici and Gierhan, 2013). Further work needs also to explore whether fibers especially of the ILF are potentially intermingled with right hemisphere fibers of the 'ATL-FFA tract', or with left hemisphere fibers of the 'ATL-VWFA' or the 'ATL-LA tract'. Such intermingled fiber trajectories might explain the inconsistent results of studies having investigated the semantic function of the ILF. Our results in healthy controls showed poor overlap of the 'ATL-VWFA tract' and of the 'ATL-LA tract' with the left ILF, and poor overlap between the 'ATL-FFA tract' and the right ILF, indicating that the explored module-hub tracts are specific fiber bundles, yet probably sharing some fiber contingents with the ILF.

Regarding the uncinate fasciculus, which links the ATL to prefrontal cortices, it remains speculative how it contributes to semantic processing given that prefrontal regions do not implement modular information. It might be that parts of the uncinate fasciculus constitute output channels conveying semantic information from the ATL to the prefrontal cortex, which subserves semantic-dependent decision making and reasoning (Shallice and Cipolotti, 2018). Conversely, some parts of the uncinate fasciculus might constitute input channels to the ATL allowing for executive-driven search, retrieval and modulation of information in the semantic system (Saur et al., 2010; Harvey et al., 2013), which has been referred to as 'semantic control' by Lambon Ralph et al. (2017).

\section{ATL tracts and SD}


Our results showing damage to the ILF and the uncinate fasciculus are in line with several previous tracking studies in SD (Galantucci et al., 2011; Agosta et al., 2013, 2015). The present study, in addition, demonstrates that tract damage in SD extends beyond these two welldocumented connections towards semantic module-hub tracts including the 'ATL-VWFA tract', the 'ATL-LA tract' and the 'ATL-FFA tract'. Furthermore, our results showed that tract alterations predominated in the left hemisphere which is consistent with the finding that early stages of SD demonstrate predominant left ATL atrophy (e.g., Mummery et al., 2000), indicating that left-lateralized damage of ATL cortices and ATL tracts is a signature of earlystage SD. Such a wide-spread ATL network damage is in line with a recent imaging study in SD (Collins et al., 2017) exploring the functional connectivity between the ATL and multiple brain regions, yet without focusing on cortical modules. Hence, it appears that the degenerative process in SD, which primarily affects the ATL (Gorno-Tempini et al., 2004, 2011), spreads insidiously to more posterior regions through the progressive erosion of the entire white matter network of semantics. As a consequence, semantic breakdown in SD patients is caused by both, damage to the ATL and to tracts conveying modular information which needs to be processed in the ATL cortices. It is, however, unclear whether the degenerative process selectively affects semantic-related networks or whether there is a general breakdown of various networks, shown to converge onto distinct sub-portions of the ATL (Papinutto et al., 2016).

\section{Limitations}

Our study has the following limitations. A first limitation is that, as in any anatomo-cognitive correlation analyses, significant correlations do not necessarily imply involvement of a given anatomical structure/tract in a cognitive process. Another limitation is related to the use of white matter tractography from diffusion MRI. It has been highlighted in many studies that tractography is highly sensitive to the parameters of image acquisition and of the image 
processing procedures (Tensaouti et al., 2011; Barrio-Arranz et al., 2015). Although we used state-of-the-art acquisition and processing procedures, we cannot rule out the possibility that some artefactual fibers have been generated by the tractography.

Finally, it remains unclear whether degenerative cortical diseases such as SD affect secondarily white matter or whether there is also cortex-independent damage of white matter/tracts. On the one hand, the absence of correlation of the left ATL with verbal semantic scores does not affect correlation results regarding the 'ATL-WFA tract' with verbal outcomes. On the other hand, the correlation of the right ATL with non-verbal scores affects correlations of tracts in the right hemisphere with non-verbal scores. This might be explained by the importance of the atrophy in the left ATL compared to the right ATL, reducing variation and thus the possibility to be correlated to semantic scores. Furthermore, it seems that verbal information is related to the 'ATL-VWFA tract' independently of the ATL or the VWFA. A hypothesis could be that the verbal information is still conveyed to the left ATL by the 'ATL-VWFA' tract, but that it is not sufficiently processed by this region due to the severe atrophy. We are therefore confident that significant correlations between tract parameters and verbal or non-verbal semantic task scores are an important indicator that these tracts, beyond the ATL cortices, contribute to semantic processing. Moreover, neuropathological investigations in the most frequent neurogenerative condition affecting the cortex, i.e. Alzheimer's disease, have indicated that white matter (tract) damage is triggered by cortical damage involving Wallerian degeneration (McAleese et al., $2015 ; 2017)$. We are aware that additional studies are needed using focal brain lesions that disrupt these particular tracts to demonstrate that they are causally involved in semantic processing.

\section{Conclusion}


Our findings reveal crucial components of the white matter network of semantics by providing evidence for three tracts and their function in conveying modular verbal and non-verbal information to the supra-modal semantic hub in the ATL. They enrich and extend the knowledge of the architecture of semantics and satisfy fundamental claims of cognitive processing models positing links between modular representations and the semantic system. Accordingly we propose to integrate modules into the currently prevailing connectionist-based 'hub-and-spokes' model while extending it to a 'hub-module-spokes' account of semantics. Our results also indicate that $\mathrm{SD}$, like most degenerative conditions, is a network disease eroding in addition to the ATL almost the entire network of verbal and non-verbal semantics. Hence both cortical and specific white matter imaging markers might be useful for monitoring disease evolution, indicating prognostic factors, and evaluating the potential efficacy of future therapy trials in SD. Our findings also open a route for future studies investigating whether the left versus right ATL cortices impact distinct module-related computations of semantics, or whether a functional lateralization of the ATL system is primarily linked to the lateralized fiber connections with left and right hemisphere modules.

\section{Acknowledgements}

This research has received funding from the program "Investissements d'avenir" ANR-10IAIHU-06 (Agence Nationale de la Recherche-10-IA Agence Institut Hospitalo-Universitaire6). O.C. is supported by a "Contrat d'Interface Local" from Assistance Publique-Hôpitaux de Paris (AP-HP). 


\section{References}

Acosta-Cabronero J, Patterson K, Fryer TD, Hodges JR, Pengas G, Williams GB, et al. Atrophy, hypometabolism and white matter abnormalities in semantic dementia tell a coherent story. Brain 2011; 134: 2025-2035.

Agosta F, Henry RG, Migliaccio R, Neuhaus J, Miller BL, Dronkers NF, et al. Language networks in semantic dementia. Brain 2010; 133(1): 286-299.

Agosta F, Galantucci S, Canu E, Cappa SF, Magnani G, Franceschi M, et al. Disruption of structural connectivity along the dorsal and ventral language pathways in patients with nonfluent and semantic variant primary progressive aphasia: a DT MRI study and a literature review. Brain Lang. 2013; 127: 157-166.

Agosta F, Ferraro PM, Canu E, Copetti M, Galantucci S, Magnani G, et al. Differentiation between Subtypes of Primary Progressive Aphasia by Using Cortical Thickness and DiffusionTensor MR Imaging Measures. Radiology 2015; 276(1): 219-227.

Avants BB, Epstein CL, Grossman M, Gee JC. Symmetric diffeomorphic image registration with cross-correlation: evaluating automated labeling of elderly and neurodegenerative brain. Med. Image Anal. 2008; 12: 26-41.

Barrio-Arranz G, de Luis-García R, Tristán-Vega A, Martín-Fernández M, Aja-Fernández S. Impact of MR Acquisition Parameters on DTI Scalar Indexes: A Tractography Based Approach . PLoS One 2015; 10

Behrens TEJ, Woolrich MW, Jenkinson M, Johansen-Berg H, Nunes RG, Clare S, et al. Characterization and propagation of uncertainty in diffusion-weighted MR imaging. Magn. Reson. Med. 2003; 50: 1077-1088.

Binder JR, Desai RH, Graves WW, Conant LL. Where Is the Semantic System? A Critical Review and Meta-Analysis of 120 Functional Neuroimaging Studies. Cereb. Cortex 2009; 19(12): 2767-2796. 
Binney RJ, Parker GJM, Lambon Ralph MA. Convergent connectivity and graded specialization in the rostral human temporal lobe as revealed by diffusion-weighted imaging probabilistic tractography. J. Cogn. Neurosci. 2012; 24: 1998-2014.

Bouhali F, Thiebaut de Schotten M, Pinel P, Poupon C, Mangin J-F, Dehaene S, et al. Anatomical connections of the visual word form area. J. Neurosci. 2014; 34: 15402-15414.

Bozeat S, Lambon Ralph MA, Patterson K, Garrard P, Hodges JR. Non-verbal semantic impairment in semantic dementia. Neuropsychologia 2000; 38: 1207-1215.

Calamante F, Tournier J-D, Jackson GD, Connelly A. Track-density imaging (TDI): superresolution white matter imaging using whole-brain track-density mapping. NeuroImage 2010; 53: $1233-1243$.

Caramazza A. How Many Levels of Processing Are There in Lexical Access? Cogn. Neuropsychol. 1997; 14: 177-208.

Caramazza A, Miozzo M. The relation between syntactic and phonological knowledge in lexical access: evidence from the 'tip-of-the-tongue' phenomenon. Cognition 1997; 64(3): 30943.

Cardebat D, Doyon B, Puel M, Goulet P, Joanette Y. [Formal and semantic lexical evocation in normal subjects. Performance and dynamics of production as a function of sex, age and educational level]. Acta Neurol. Belg. 1990; 90: 207-217.

Catani M, Howard RJ, Pajevic S, Jones DK. Virtual in vivo interactive dissection of white matter fasciculi in the human brain. NeuroImage 2002; 17: 77-94.

Catani M, Jones DK, Donato R, Ffytche DH. Occipito-temporal connections in the human brain. Brain 2003; 126(9): 2093-2107.

Cohen L, Dehaene S, Naccache L, Lehéricy S, Dehaene-Lambertz G, Hénaff MA, et al. The visual word form area: spatial and temporal characterization of an initial stage of reading in normal subjects and posterior split-brain patients. Brain 2000; 123(2): 291-307. 
Cohen L, Lehéricy S, Chochon F, Lemer C, Rivaud S, Dehaene S. Language-specific tuning of visual cortex? Functional properties of the Visual Word Form Area. Brain 2002; 125(5): 10541069.

Collins JA, Montal V, Hochberg D, Quimby M, Mandelli ML, Makris N, et al. Focal temporal pole atrophy and network degeneration in semantic variant primary progressive aphasia. Brain 2017; 140(2): 457-471.

Damasio H, Tranel D, Grabowski T, Adolphs R, Damasio A. Neural systems behind word and concept retrieval. Cognition 2004; 92: 179-229.

Deloche G, Metz-Lutz M, Kremin H. Test de dénomination orale de 80 images. In: Editions du Centre de Psychologie Appliquée CDP. Paris: 1997.

Dubois B, Slachevsky A, Litvan I, Pillon B. The FAB: a Frontal Assessment Battery at bedside. Neurology 2000; 55: 1621-1626.

Duffau H. The anatomo-functional connectivity of language revisited. New insights provided by electrostimulation and tractography. Neuropsychologia 2008; 46: 927-934.

Duffau H, Gatignol P, Mandonnet E, Peruzzi P, Tzourio-Mazoyer N, Capelle L. New insights into the anatomo-functional connectivity of the semantic system: a study using corticosubcortical electrostimulations. Brain 2005; 128(4): 797-810.

Fischl B. FreeSurfer. 20 Years of fMRI, NeuroImage 2012; 62: 774-781.

Fodor, JA. (1983). The Modularity of Mind. Cambridge, Mass., MIT Press.

Folstein MF, Folstein SE, McHugh PR. 'Mini-mental state'. A practical method for grading the cognitive state of patients for the clinician. J. Psychiatr. Res. 1975; 12: 189-198.

Friederici AD, Gierhan SME. The language network. Curr. Opin. Neurobiol. 2013; 23: 250254.

Gainotti G. Laterality effects in normal subjects' recognition of familiar faces, voices and names. Perceptual and representational components. Neuropsychologia 2013; 51: 1151-1160. 
Galantucci S, Tartaglia MC, Wilson SM, Henry ML, Filippi M, Agosta F, et al. White matter damage in primary progressive aphasias: a diffusion tensor tractography study. Brain 2011; 134(10): 3011-3029.

Gauthier I, Tarr MJ, Moylan J, Anderson AW, Skudlarski P, Gore JC. Does visual subordinatelevel categorisation engage the functionally defined fusiform face area? Cogn. Neuropsychol. 2000; 17: 143-164.

Gerlach C, Law I, Gade A, Paulson OB. Perceptual differentiation and category effects in normal object recognition: a PET study. Brain 1999; 122(11): 2159-2170.

Gorno-Tempini ML, Dronkers NF, Rankin KP, Ogar JM, Phengrasamy L, Rosen HJ, et al. Cognition and Anatomy in Three Variants of Primary Progressive Aphasia. Ann. Neurol. 2004; 55: $335-346$.

Gorno-Tempini ML, Hillis AE, Weintraub S, Kertesz A, Mendez M, Cappa SF, et al. Classification of primary progressive aphasia and its variants. Neurology 2011; 76(11): 10061014.

Graves WW, Grabowski TJ, Mehta S, Gordon JK. A neural signature of phonological access: distinguishing the effects of word frequency from familiarity and length in overt picture naming. J. Cogn. Neurosci. 2007; 19: 617-631.

Graves WW, Grabowski TJ, Mehta S, Gupta P. The left posterior superior temporal gyrus participates specifically in accessing lexical phonology. J. Cogn. Neurosci. 2008; 20: 16981710.

Guo CC, Gorno-Tempini ML, Gesierich B, Henry M, Trujillo A, Shany-Ur T, et al. Anterior temporal lobe degeneration produces widespread network-driven dysfunction. Brain 2013 136(10): 2979-2991. 
Harvey DY, Wei T, Ellmore TM, Hamilton AC, Schnur TT. Neuropsychological evidence for the functional role of the uncinate fasciculus in semantic control. Neuropsychologia 2013; 51: 789-801.

Hickok G, Poeppel D. Dorsal and ventral streams: a framework for understanding aspects of the functional anatomy of language. Cognition 2004; 92: 67-99.

Hocking RR. (1985). The analysis of linear models. Brooks/Cole Pub Co.

Howard D, Patterson KE, Company TVT. The pyramids and palm trees test : a test of semantic access from words and pictures. Bury St Edmunds: Thames Valley Test Company; 1992.

Iaccarino L, Crespi C, Della Rosa PA, Catricalà E, Guidi L, Marcone A, et al. The semantic variant of primary progressive aphasia: Clinical and neuroimaging evidence in single subjects. PLoS ONE 2015; 10: 1-17.

Indefrey P, Levelt WJM. The spatial and temporal signatures of word production components. Cognition 2004; 92: 101-144.

Jeurissen B, Tournier J-D, Dhollander T, Connelly A, Sijbers J. Multi-tissue constrained spherical deconvolution for improved analysis of multi-shell diffusion MRI data. NeuroImage 2014; 103: 411-426.

Jezzard P, Balaban RS. Correction for geometric distortion in echo planar images from B0 field variations. Magn. Reson. Med. 1995; 34: 65-73.

Jouen AL, Ellmore TM, Madden-Lombardi CJ, Pallier C, Dominey PF, Ventre-Dominey J. Beyond the word and image: II- Structural and functional connectivity of a common semantic system. NeuroImage 2018; 166: 185-197.

Kanwisher N, McDermott J, Chun MM. The fusiform face area: a module in human extrastriate cortex specialized for face perception. J. Neurosci. 1997; 17: 4302-4311.

Kanwisher N, Yovel G. The fusiform face area: a cortical region specialized for the perception of faces. Philos. Trans. R. Soc. B Biol. Sci. 2006; 361: 2109-2128. 
Kloke, J. D., \& McKean, J. W. (2012). Rfit: Rank-based Estimation for Linear Models. $R$ Journal, 4(2).

Kotz SA, Cappa SF, von Cramon DY, Friederici AD. Modulation of the lexical-semantic network by auditory semantic priming: an event-related functional MRI study. NeuroImage 2002; 17: 1761-1772.

Laisney M, Eustache F, Desgranges B, Libbey J, Revue E. Évaluation de la mémoire sémantique relative aux personnes célèbres - SemPer Assessment of semantic memory for famous people - SemPer. Rev. Neuropsychol. 2009; 1: 175-183.

Lambon Ralph MA, Graham KS, Patterson K, Hodges JR. Is a picture worth a thousand words? Evidence from concept definitions by patients with semantic dementia. Brain Lang. 1999; 70: 309-335.

Lambon Ralph MA, Jefferies E, Patterson K, Rogers TT. The neural and computational bases of semantic cognition. Nat Rev Neurosci. 2017; 18(1): 42-55.

Leemans A, Jones DK. The B-matrix must be rotated when correcting for subject motion in DTI data. Magn. Reson. Med. 2009; 61: 1336-1349.

Levelt WJ, Roelofs A, Meyer AS. A theory of lexical access in speech production. Behav. Brain Sci. 1999; 22: 1-38.

Lloyd-Jones TJ, Humphreys GW. Perceptual differentiation as a source of category effects in object processing: evidence from naming and object decision. Mem. Cognit. 1997; 25: 18-35. Makris N, Papadimitriou GM, Kaiser JR, Sorg S, Kennedy DN, Pandya DN. Delineation of the Middle Longitudinal Fascicle in Humans: A Quantitative, In Vivo, DT-MRI Study. Cereb. Cortex 2009; 19: 777-785.

Makris N, Preti MG, Asami T, Pelavin P, Campbell B, Papadimitriou GM, et al. Human middle longitudinal fascicle: variations in patterns of anatomical connections. Brain Struct. Funct. 2013; 218: 951-968. 
Makris N, Zhu A, Papadimitriou GM, Mouradian P, Ng I, Scaccianoce E, et al. Mapping temporo-parietal and temporo-occipital cortico-cortical connections of the human middle longitudinal fascicle in subject-specific, probabilistic, and stereotaxic Talairach spaces. Brain Imaging Behav. 2017; 11: 1258-1277.

Mandonnet E, Nouet A, Gatignol P, Capelle L, Duffau H. Does the left inferior longitudinal fasciculus play a role in language? A brain stimulation study. Brain 2007; 130: 623-629.

Mattis S. Mental status examination for organic mental syndrome in elderly patients. In: Bellak L, Karasu TB, editor(s). New York: Grune \& Straton; 1976. p. 77-121.

Mazaux and Orgogozo. Boston Diagnostic Aphasia Examination; Version française. 1982.

McAleese KE, Firbank M, Dey M, Colloby SJ, Walker L, Johnson M, et al. Cortical tau load is associated with white matter hyperintensities. Acta Neuropathol Commun. 2015; 30; 3:60.

McAleese KE, Walker L, Graham S, Moya ELJ, Johnson M, Erskine D, et al. Parietal white matter lesions in Alzheimer's disease are associated with cortical neurodegenerative pathology, but not with small vessel disease. Acta Neuropathol. 2017; 134(3): 459-473.

McCandliss BD, Cohen L, Dehaene S. The visual word form area: expertise for reading in the fusiform gyrus. Trends Cogn. Sci. 2003; 7: 293-299.

Merck C, Charnallet A, Auriacombe S, Belliard S, Hahn-Barma V, Kremin H, et al. La batterie d'évaluation des connaissances sémantiques du GRECO (BECS-GRECO): validation et données normatives. Rev. Neuropsychol. 2011; 3: 235.

Mesulam MM, Wieneke C, Hurley R, Rademaker A, Thompson CK, Weintraub S, et al. Words and objects at the tip of the left temporal lobe in primary progressive aphasia. Brain $2013 ; 136$ : 601-618.

Mion M, Patterson K, Acosta-cabronero J, Pengas G, Izquierdo-garcia D, Hong YT, et al. What the left and right anterior fusiform gyri tell us about semantic memory. Brain 2010; 133(11): $3256-3268$. 
Mori S, Wakana S, Nagae-Poetscher L, van Zijl P. MRI Atlas of Human White Matter. Amsterdam: Elsevier; 2005.

Mummery CJ, Patterson K, Price CJ, Ashburner J, Frackowiak RSJ, Hodges JR. A voxel-based morphometry study of semantic dementia: Relationship between temporal lobe atrophy and semantic memory. Ann. Neurol. 2000; 47: 36-45.

Operto G, Chupin M, Batrancourt B, Habert MO, Colliot O, Benali H, et al.; CATI Consortium. CATI: A Large Distributed Infrastructure for the Neuroimaging of Cohorts. Neuroinformatics. 2016; 14(3): 253-64.

Papinutto N, Galantucci S, Mandelli ML, Gesierich B, Jovicich J, Caverzasi E, et al. Structural connectivity of the human anterior temporal lobe: a diffusion magnetic resonance imaging study. Hum. Brain Mapp. 2016; 37: 2210-2222.

Parker GJM, Luzzi S, Alexander DC, Wheeler-Kingshott CAM, Ciccarelli O, Lambon Ralph MA. Lateralization of ventral and dorsal auditory-language pathways in the human brain. NeuroImage 2005; 24: 656-666.

Pascual B, Masdeu JC, Hollenbeck M, Makris N, Insausti R, Ding S-L, et al. Large-scale brain networks of the human left temporal pole: a functional connectivity MRI study. Cereb. Cortex 2015; 25(3): 680-702.

Patterson K, Nestor PJ, Rogers TT. Where do you know what you know? The representation of semantic knowledge in the human brain. Nat. Rev. Neurosci. 2007; 8: 976-987.

Pinker S, Jackendoff R. The faculty of language: what's special about it? Cognition 2005; 95: 201-236.

Pobric G, Jefferies E, Ralph MAL. Anterior temporal lobes mediate semantic representation: mimicking semantic dementia by using rTMS in normal participants. Proc. Natl. Acad. Sci. U. S. A. 2007; 104: 20137-20141. 
Pobric G, Jefferies E, Ralph MAL. Amodal semantic representations depend on both anterior temporal lobes: evidence from repetitive transcranial magnetic stimulation. Neuropsychologia 2010; 48: 1336-1342.

R Core Team. R: A Language and Environment for Statistical Computing. Vienna, Austria: R Foundation for Statistical Computing; 2013.

Race DS, Hillis AB. Naming. In: Toga AW, editor(s). Brain Mapping. Waltham: Academic Press; 2015. p. 671-675.

Raven J, Raven JC, Court JH. Raven manual: Standard progressive matrices. Oxford, England: Oxford Psychologists Press. 1998.

Rice GE, Lambon Ralph MA, Hoffman P. The Roles of Left Versus Right Anterior Temporal Lobes in Conceptual Knowledge: An ALE Meta-analysis of 97 Functional Neuroimaging Studies. Cereb. Cortex 2015; 25: 4374-4391.

Rice GE, Hoffman P, Lambon Ralph MA. Graded specialization within and between the anterior temporal lobes. Ann N Y Acad Sci. 2015; 1359: 84-97.

Saur D, Kreher BW, Schnell S, Kummerer D, Kellmeyer P, Vry M-S, et al. Ventral and dorsal pathways for language. Proc. Natl. Acad. Sci. 2008; 105: 18035-18040.

Saur D, Schelter B, Schnell S, Kratochvil D, Küpper H, Kellmeyer P, et al. Combining functional and anatomical connectivity reveals brain networks for auditory language comprehension. NeuroImage 2010; 49: 3187-3197.

Shallice T, Cipolotti L. The Prefrontal Cortex and Neurological Impairments of Active Thought. Annu. Rev. Psychol. 2018; 69: 157-180.

Snowden JS, Thompson JC, Neary D. Knowledge of famous faces and names in semantic dementia. Brain 2004; 127: 860-872.

Tarr MJ, Gauthier I. FFA: a flexible fusiform area for subordinate-level visual processing automatized by expertise. Nat. Neurosci. 2000; 3: 764-769. 
Tensaouti F, Lahlou I, Clarisse P, Lotterie JA, Berry I. Quantitative and reproducibility study of four tractography algorithms used in clinical routine. J Magn Reson Imaging 2011; 34: 165172.

Tournier J-D, Calamante F, Connelly A. Robust determination of the fibre orientation distribution in diffusion MRI: non-negativity constrained super-resolved spherical deconvolution. NeuroImage 2007; 35: 1459-1472.

Tournier J-D, Calamante F, Connelly A. MRtrix: Diffusion tractography in crossing fiber regions. Int. J. Imaging Syst. Technol. 2012; 22: 53-66.

Tustison NJ, Avants BB. Explicit B-spline regularization in diffeomorphic image registration. Front. Neuroinformatics 2013; 7: 39.

Tyler LK, Marslen-Wilson WD, Stamatakis EA. Differentiating lexical form, meaning, and structure in the neural language system. Proc. Natl. Acad. Sci. U S A 2005; 102(23): 83758380.

Ullman MT. A neurocognitive perspective on language: the declarative/procedural model. Nat Rev Neurosci. 2001; 2(10): 717-726.

Vandenberghe R, Price C, Wise R, Josephs O, Frackowiak RS. Functional anatomy of a common semnatic system for words and pictures. Nature 1996; 383: 254-256.

Vandenberghe R, Wang Y, Nelissen N, Vandenbulcke M, Dhollander T, Sunaert S, et al. The associative-semantic network for words and pictures: effective connectivity and graph analysis. Brain Lang. 2013; 127: 264-272.

$\mathrm{Xu}$ Y. Revisiting the role of the fusiform face area in visual expertise. Cereb. Cortex 2005; 15 : $1234-1242$. 


\section{Figure Legends}

Figure 1 Cortical regions used for fiber tracking: i) the ATL hub (yellow) and ii) the three module-implementing areas: FFA (red), VWFA (blue), lexical-related area ('Lexicon Area') in posterior/superior temporal cortices (green).

Left hemisphere

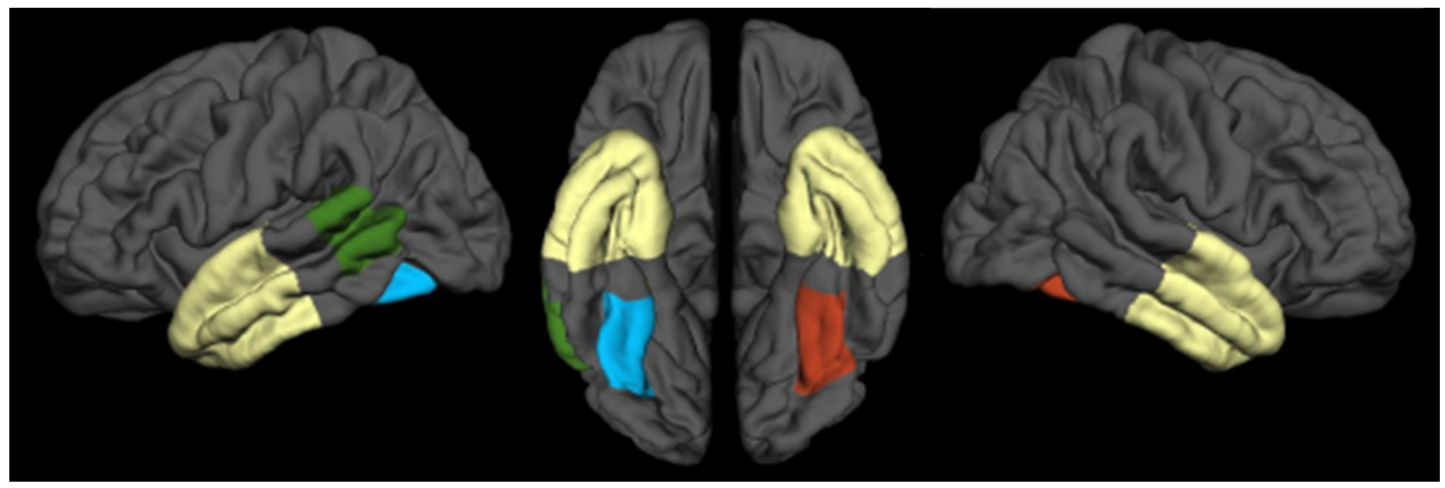

Right hemisphere 
Figure 2 Semantic performance for the verbal and non-verbal/visual modality in SD patients and healthy controls (mean values \pm standard deviations)

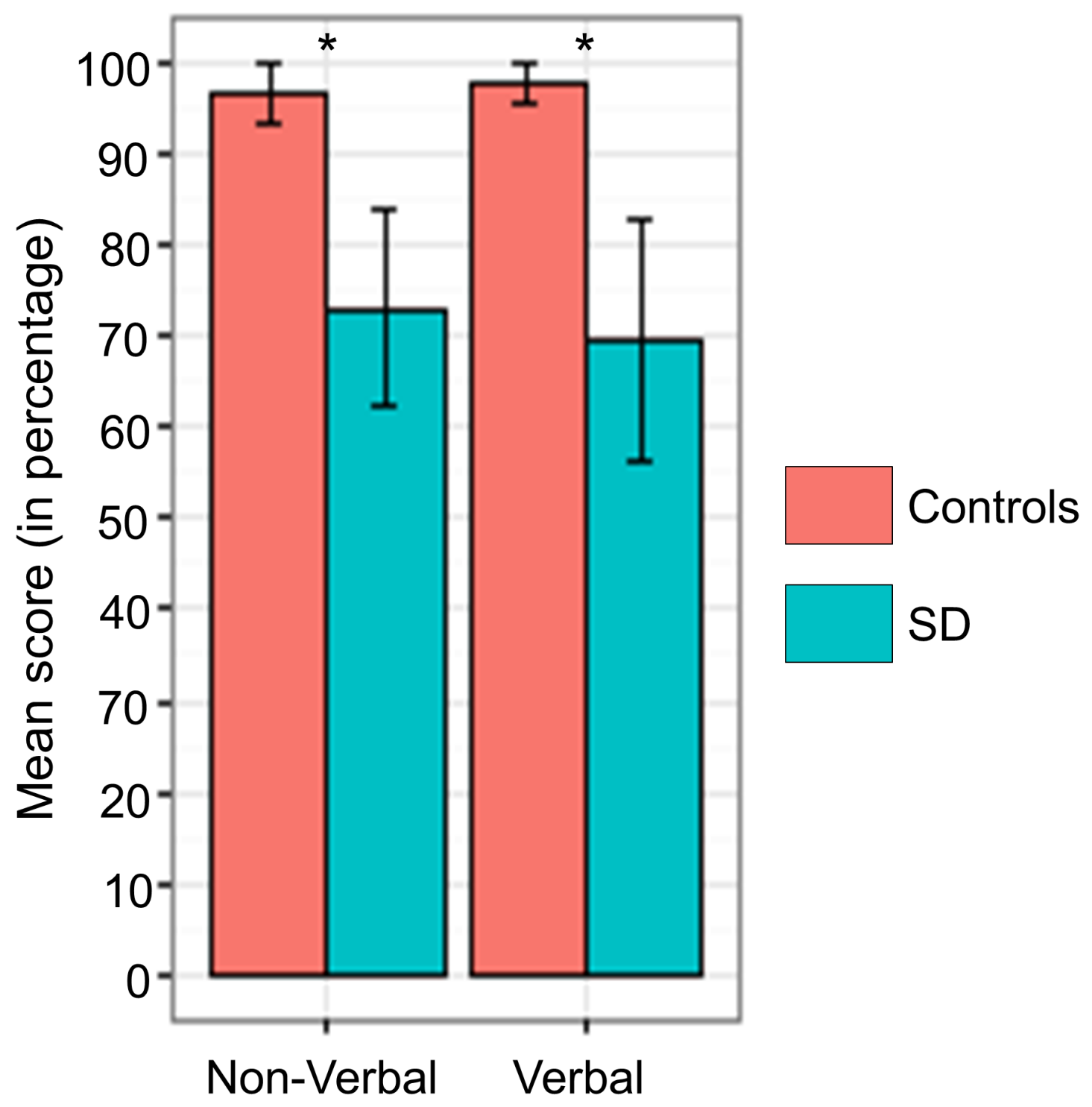


Figure 3 Brain regions with significantly reduced cortical thickness in SD patients as compared to healthy controls. Corrected p-values for cluster correction are displayed with a blue colormap.

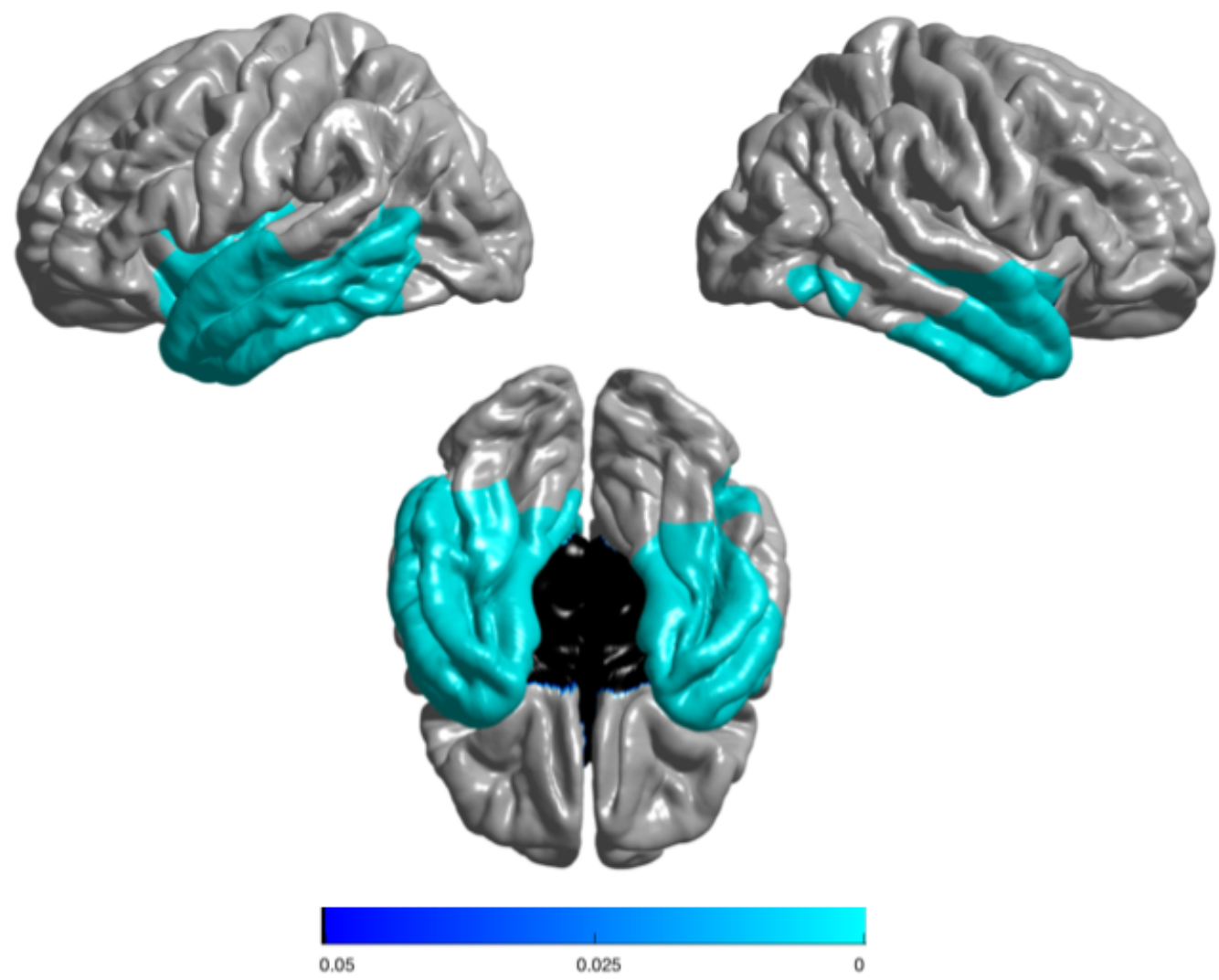


Figure 4 Tracts connecting the ATL to the FFA (red), to the VWFA (blue), and to the 'Lexicon Area' in posterior/superior temporal cortices (green).
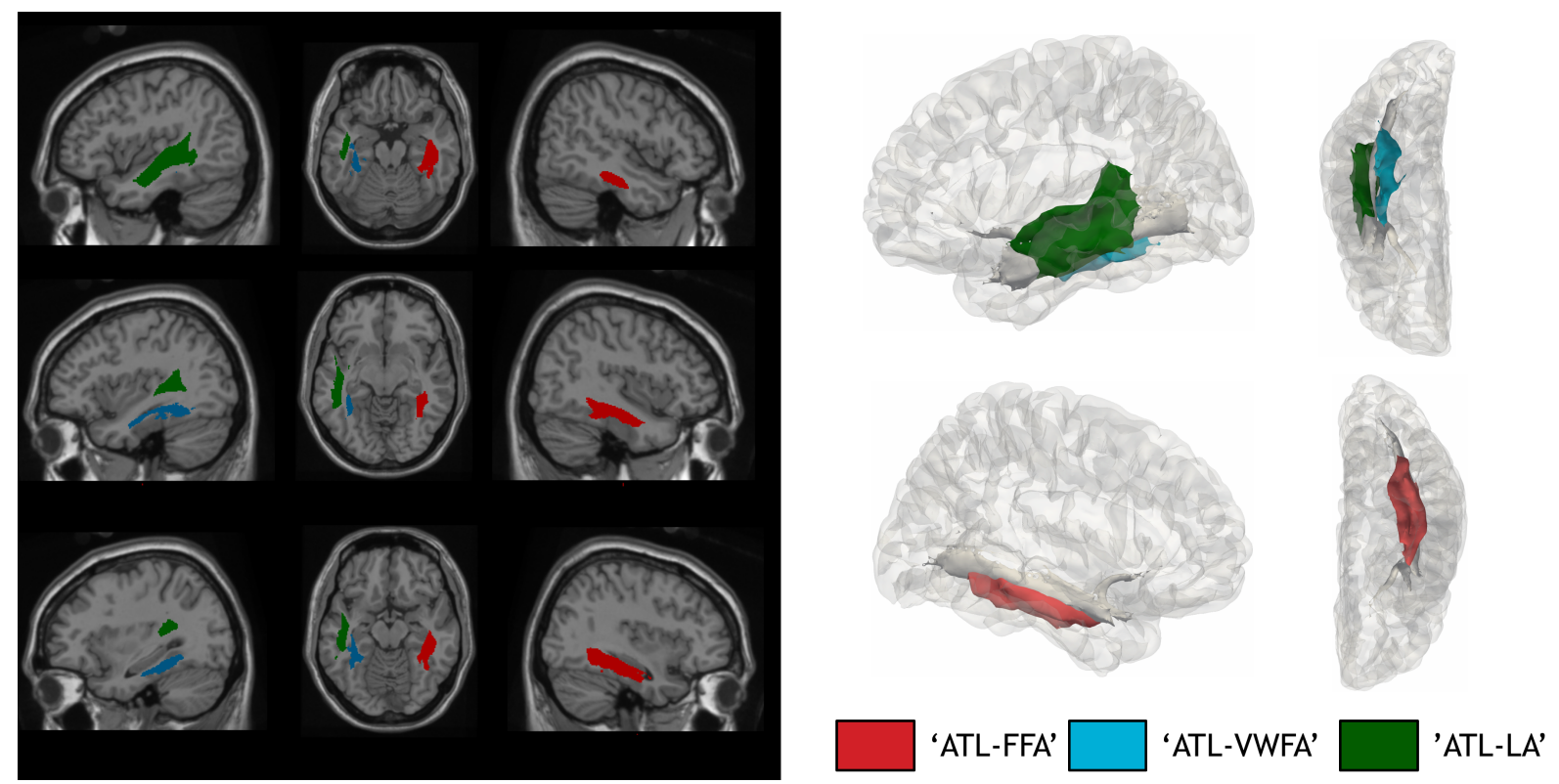
Supplementary Figure The correlation circle: correlations between the different MRI measures and semantic scores and the two first principal components via coordinates. The two first components sum up $68 \%$ of the total variance.

Semantic composite (verbal, non-verbal) scores are measured in percentage. L indicates left hemisphere, $\mathrm{R}$ indicates right hemisphere. Mean Diffusivity (MD) for white matter tracts are: UNC-L, UNC-R, ILF-L, ILF-R, ATL-WVFA-L, ATL-FFA-R, ATL-LA-L, and ATL-LA-R. Mean cortical thickness of the regions of interests are: ATL-L, ATL-R, WVFA-L, FFA-R, LAL, and LA-R.

\section{Variables factor map (PCA)}

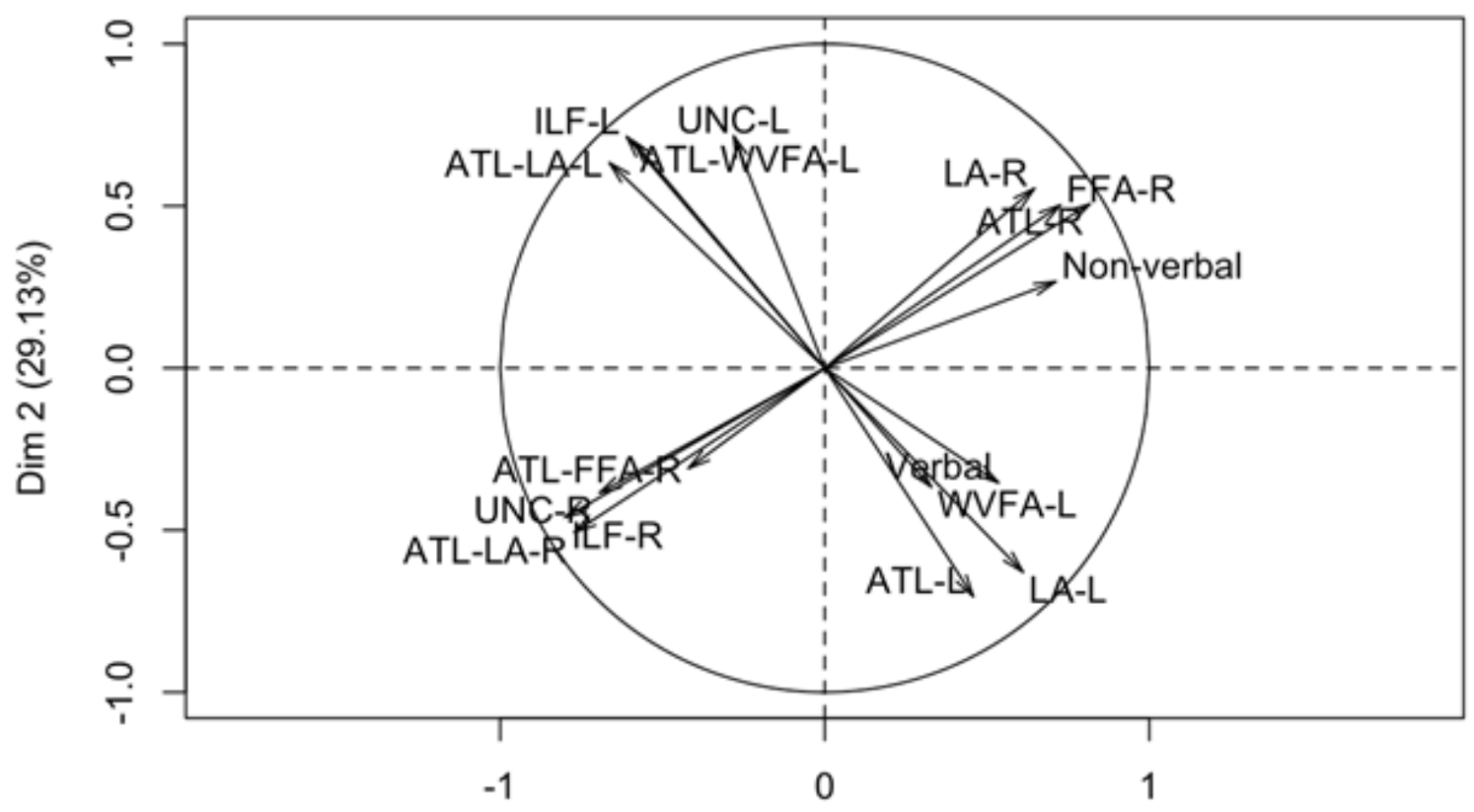

$\operatorname{Dim} 1(38.91 \%)$ 